Boletín de la Sociedad Geológica Mexicana

VOLUMEN 62, NÚM. 3, 2010, P. 359-377

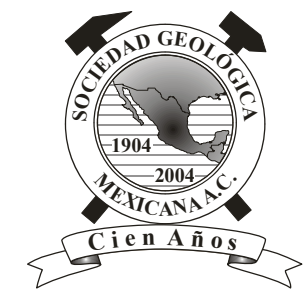

\title{
Evidencias de cambio climático y ambiental en registros glaciales y en cuencas lacustres del centro de México durante el último máximo glacial
}

\author{
Margarita Caballero ${ }^{1, *}$, Socorro Lozano-García ${ }^{2}$, Lorenzo Vázquez-Selem³, Beatriz Ortega ${ }^{1}$ \\ ${ }^{1}$ Instituto de Geofísica, Universidad Nacional Autónoma de México, 04510, México D. F., México. \\ ${ }^{2}$ Instituto de Geología, Universidad Nacional Autónoma de México, 04510, México D. F., México. \\ ${ }^{3}$ Instituto de Geografía, Universidad Nacional Autónoma de México, 04510, México D. F., México. \\ *maga@geofisica.unam.mx
}

\begin{abstract}
Resumen
Se presenta una revisión de la información disponible sobre historia glacial, cambios en los niveles lacustres y datos sobre paleovegetación del Pleistoceno tardío de varios volcanes y cuencas localizadas en la Faja Volcánica Transmexicana (FVTM), permitiendo establecer el estado del conocimiento sobre el cambio climático durante el último máximo glacial (UMG) en el centro de México. Los datos se presentan en cuatro ventanas de tiempo: Glacial Temprano (GTM): 25000-22000 cal AP; Último Máximo Glacial (UMG): 22000-18000 cal AP; Glacial Tardío (GTA): 18000-15000 cal AP y Glacial Terminal (GTE): 15000-12000 cal AP. Las evidencias geomorfológicas del Pleistoceno tardío del volcán Iztaccíhuatl ofrecen el registro más completo de avances glaciales de los últimos 30000 años; en otras elevaciones el registro glacial es fragmentario (debido al vulcanismo) o más limitado (debido a la menor altitud). Durante el GTM no hay evidencias de avances glaciales pero los datos de las cuencas lacustres indican niveles lacustres bajos. Para el UMG se estima una disminución en la temperatura de 6 a $8^{\circ} \mathrm{C}$, con la línea de las nieves descendiendo hasta $3940 \mathrm{~m}$ en el volcán Iztaccíhuatl, a $3400 \mathrm{~m}$ en el Tancítaro y a $3650 \mathrm{~m}$ en el Cofre de Perote. Con base en los conjuntos polínicos se propone un desplazamiento del límite bosque de Pinus-zacatonal alpino durante el UMG de $\sim 1000 \mathrm{~m}$ asociado a cambios en la distribución y en la composición de la vegetación. Durante este periodo hay evidencias de niveles lacustres bajos en las cuencas de México y del Lerma, mientras que en el sector occidental de la FVTM el registro de Pátzcuaro ha sido interpretado como una etapa de nivel lacustre alto siendo que para Zacapu-Cuitzeo y Zirahuén los registros indican niveles lacustres bajos. Se infiere la existencia de un gradiente de humedad de las costas hacia el interior de la FVTM. Para el GTA las evidencias de retroceso en los glaciares los sitúan tan solo 100 m por arriba del UMG en el Iztaccíhuatl. Al inicio del GTE los glaciares retroceden lentamente y en pulsos, y después de 14000 cal AP de manera pronunciada; en algunos de los lagos hay hiatos en la sedimentación, como en Tecocomulco, Texcoco, Cuitzeo y Zacapu, indicando condiciones secas.
\end{abstract}

Palabras clave: Glacial tardío, centro de México, historia glacial, paleolimnología, palinología, Faja Volcánica Transmexicana.

\begin{abstract}
Significant information is available regarding glacial history, lake level and vegetation history from several basins and volcanoes during the late Pleistocene in the Transmexican Volcanic Belt (TMVB). Here we present a synthesis and compilation of that information to establish the climatic conditions in this region during the Last Glacial Maximum. The data is organized in four time windows: Early Glacial: 25000-22000 cal BP, Last Glacial Maximum: 22000-18000 cal BP, Late Glacial: 18000-15000 cal BP and Terminal Glacial 15000-12000 cal BP. The late Pleistocene geomorphological evidence from Iztaccihuatl volcano offer the most complete record of glacial advances during the last $30000 \mathrm{yr}$; at other elevations the glacial history is fragmentary (due to volcanic activity) or more
\end{abstract}


limited (due to lower altitudes). For the Early Glacial no evidence of glacial advance is recorded but data from the lacustrine basins indicate low lake levels. During the Last Glacial Maximum a decrease in temperature of 6 to $8^{\circ} \mathrm{C}$ is estimated, and the equilibrium line altitude is reconstructed at $3940 \mathrm{~m}$ for Iztaccihuatl, $3400 \mathrm{~m}$ for Tancitaro, and $3650 \mathrm{~m}$ for Cofre de Perote. A downward displacement of $\sim 1000 \mathrm{~m}$ for the limit of Pine forests with alpine vegetation, as well as changes in vegetation composition and distribution, are documented for the Late Glacial Maximum on the basis of palynological data. During this period low lake levels are recorded in the lakes of the Mexico and Lerma basins, while in the western sector of the TMVB most of the basins show low lake levels (Zirahuen, Zacapu and Cuitzeo) and only the record of Lake Patzcuaro suggests high stands for this period. Based on the synthesis of the data, a humidity gradient from the coasts towards the interior of the TMVB is inferred. For the Late Glacial period, a glacier retreat of $\sim 100$ $m$ compared to the Last Glacial Maximum is detected at Iztaccihuatl volcano. At the beginning of the Terminal Glacial all the glaciers retreated slowly and intermittently, then markedly after 14000 cal BP. At several lakes such as Tecocomulco, Texcoco, Cuitzeo and Zacapu sedimentation hiatus are recorded, indicating drier conditions.

Keywords: Late Glacial, central Mexico, glacial history, paleolimnology, palynology, Transmexican Volcanic Belt.

\section{Introducción}

Durante los últimos 400000 años el clima en la Tierra se ha caracterizado por tener fluctuaciones muy marcadas en ciclos de $\sim 100000$ años. Estos ciclos se manifiestan con la alternancia de etapas más frías que el clima actual (en promedio, $8^{\circ} \mathrm{C}$ menos), conocidas como glaciales, y etapas en las que el clima es similar o un poco más cálido $\left(2^{\circ}\right.$ a 3 ${ }^{\circ} \mathrm{C}$ mayor) que el presente, conocidas como interglaciales. Durante los periodos glaciales, los grandes casquetes de hielo del planeta se expanden hacia posiciones mucho más ecuatoriales que las actuales y, en consecuencia, desciende el nivel del mar.

El llamado Último Máximo Glacial (UMG, Last Glacial Maximum) representa el momento más reciente en el que los grandes glaciares alcanzaron sus máximos volúmenes (Mix et al., 2001). Este periodo, fechado entre 26500 y $20000-$ $19000 \mathrm{cal}$ AP (años calendarizados antes del presente), se caracterizó también por un descenso en el nivel del mar de $\sim 130 \mathrm{~m}$ en relación al actual (Clark et al., 2009). En glaciares pequeños, como los de montaña, la máxima expansión no fue simultánea a la de los grandes casquetes, ni ocurrió sincrónicamente en todas partes del planeta. Incluso varios glaciares de montaña presentaron un máximo antes que los grandes casquetes (Gillespie y Molnar, 1995). De allí que exista confusión en el uso del término UMG, ya que en ocasiones se refiere al máximo local de estos pequeños glaciares y en otras al máximo de los grandes casquetes, al que se podría referir como el UMG planetario.

Es relevante indagar sobre la naturaleza de los cambios ambientales ocurridos durante el UMG porque representa un estado del clima global dramáticamente diferente al actual, que mantuvo un relativo equilibrio durante varios milenios, y por ello puede servir para evaluar la sensibilidad de la respuesta de los sistemas ambientales a condiciones climáticas extremas y definir los mecanismos responsables del cambio climático (Mix et al., 2001).

A partir de la reconstrucción de la temperatura superficial del mar durante el UMG, que realizó el proyecto CLIMAP (1981), fueron evidentes muchas inconsistencias con los registros de los trópicos, en particular con las evidencias de glaciación tropical, que indicaban una depresión de la temperatura mucho mayor a la que se reconstruía para el océano (Rind y Peteet, 1985). Aunque estudios posteriores han estimado enfriamientos aún mayores en los trópicos (p. e., Lea et al., 2000), falta aún determinar la magnitud del enfriamiento en los continentes y su impacto ambiental. La compilación de Farrera et al. (1999) muestra que hay diferencias importantes a este respecto entre diversas zonas tropicales del mundo y que los trópicos americanos se encuentran entre las zonas con mayor depresión de la temperatura durante el UMG. La variación de la precipitación es también un aspecto poco conocido durante esta fase. En general, los registros existentes apuntan hacia una mayor aridez tropical, con niveles lacustres bajos registrados en regiones como el África tropical. Esto ha sido interpretado como indicador de que durante el UMG los elementos de la circulación atmosférica global, como las celdas de alta presión subtropical (CAPS) y la zona intertropical de convergencia (ZITC) estaban desplazados hacia el sur de su posición actual y que los sistemas monzónicos estaban deprimidos, favoreciendo climas tropicales más secos (Kutzbach et al., 1993). Sin embargo, este patrón no es universal y en algunas regiones tropicales parecen haber existido condiciones locales relativamente húmedas.

Estos aspectos - la magnitud del enfriamiento, la disminución/aumento relativo de la humedad durante el UMG - también están pendientes de ser resueltos en el centro de México (Metcalfe et al., 2000; Metcalfe, 2006). Mientras que diversos registros de las cuencas interiores sugieren mayor aridez (Caballero et al., 1999; LozanoGarcía et al., 2005), para el centro-poniente se han propuesto condiciones relativamente húmedas (Bradbury, 1997, 2000) y recientemente también para el sur de la península de Yucatán (Hodell et al., 2008; Bush et al., 2009).

En este contexto, el presente trabajo presenta una revisión del estado del conocimiento sobre el clima y el ambiente del centro de México durante el UMG, partiendo del hecho de que la información disponible para este periodo es aún fragmentaria y limitada a pocas localidades, algunas 
de ellas con problemas como la pobre preservación del registro o el intenso impacto por la actividad volcánica de la zona. El objetivo central de este trabajo es comparar los registros glacial-geomorfológicos, palinológicos y paleolimnológicos que cubren el Pleistoceno tardío (25000 - 12000 años AP), con énfasis en el UMG, a lo largo de los volcanes y las cuencas de altura que se ubican en la Faja Volcánica Transmexicana (FVTM). Al comparar información provista por diferentes registros es posible, por un lado, responder algunas de las cuestiones aún no resueltas $\mathrm{y}$, por otro lado, mostrar las inconsistencias y las zonas y fases donde es necesaria mayor investigación. Cada tipo de registro ofrece información paleoclimática distinta y a menudo complementaria. Los registros paleolimnológicos provienen de sedimentos lacustres y con frecuencia los datos palinológicos provienen de las mismas secuencias. Los sedimentos lacustres representan a las localidades de menor altitud dentro de cada cuenca, son ambientes con una sedimentación más o menos continua y por lo tanto contienen la mayor resolución temporal de los registros aquí considerados. Los registros glaciares provienen de las elevaciones más altas de cada cuenca y documentan los eventos de máxima extensión de hielo, de "estancamiento" durante una regresión glacial, y en algunos casos de la regresión misma. Existen otros registros paleoclimáticos que incluyen el UMG en el centro de México, como los de megafauna pleistocénica (Ferrusquía-Villafranca et al., 2010), paleosuelos (p. e., Sedov et al., 2009a) y sedimentos oceánicos (p. e., Machain-Castillo et al., en prensa). Sin embargo, por la extensión que requiere la puntual discusión de los mismos, ésta no se incluye en este trabajo. Por otra parte, menos del $3.5 \%$ de los registros de mamíferos en México cuentan con fechamientos radiométricos (Ferrusquía-Villafranca et al., 2010).

\section{Descripción de la zona}

La FVTM alberga una serie de volcanes y de cuencas de altura que se extienden en sucesión desde el golfo de México al Pacífico aproximadamente a lo largo del paralelo $20^{\circ} \mathrm{N}$ (Figura 1). El intenso volcanismo en la FVTM, que inició en el Terciario y continúa hasta el reciente ha formado los mayores edificios volcánicos del país: Pico de Orizaba, Popocatépetl, Iztaccíhuatl, Nevado de Toluca, Tancítaro, Nevado de Colima, etc. Esto ha resultado en un gradiente altitudinal de más de $5000 \mathrm{~m}$ en el centro de México.

En general el clima actual en el centro de México se caracteriza por tener una máxima precipitación en verano, cuando la ZITC alcanza su posición más norteña y la humedad es transportada por los vientos alisios desde el Golfo de México y el Caribe, y por el sistema tipo monzón que provee humedad desde el Pacífico tropical. Las tormentas tropicales y huracanes también son una fuente de humedad importante en ambas vertientes hacia finales de verano y principios del otoño. El invierno se caracteriza

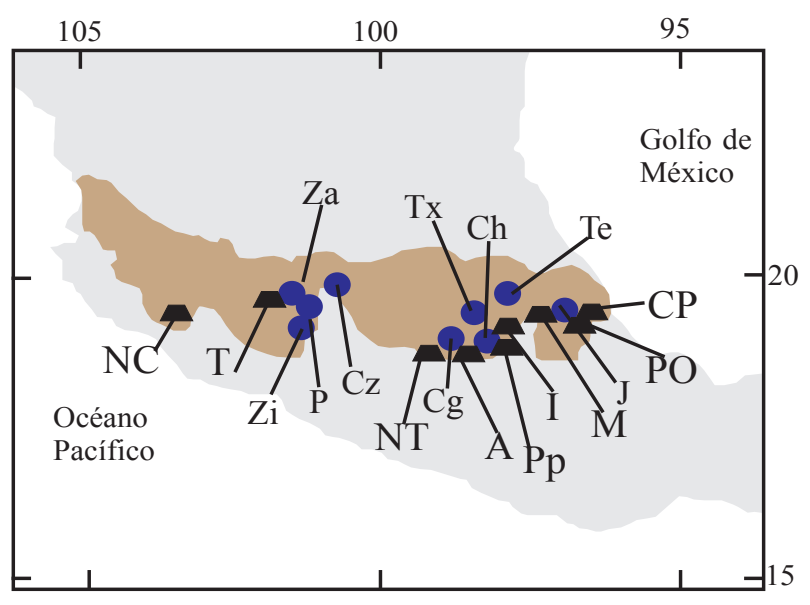

Figura 1. Mapa de localización de los sitios a lo largo de la FVTM (sombreado café) mencionados en el texto. Montañas (negro): $\mathrm{CP}=$ Cofre de Perote, $\mathrm{PO}=$ Pico de Orizaba, $\mathrm{M}=$ Malinche, $\mathrm{I}=$ Iztaccíhuatl, $\mathrm{Pp}=$ Popocatépetl $\mathrm{A}=$ Ajusco, $\mathrm{NT}=$ Nevado de Toluca, $\mathrm{T}=$ Tancítaro, $\mathrm{NC}=$ Nevado de Colima. Lagos (azul): $\mathrm{J}=$ Jalapasquillo, $\mathrm{Te}=\mathrm{Tecocomulco}, \mathrm{Ch}$ $=$ Chalco, $\mathrm{Tx}=\mathrm{Texcoco}, \mathrm{Cg}=$ Chignahuapan, $\mathrm{Cz}=$ Cuitzeo, $\mathrm{P}=$ Pátzcuaro, $\mathrm{Zi}=$ Zirahuén, $\mathrm{Za}=$ Zacapu.

por el desplazamiento de masas de aire polar sobre la Mesa Central y la vertiente del Golfo de México, asociadas con un marcado descenso en la temperatura, lo que genera que los inviernos de la parte central y la vertiente del Golfo de México sean levemente más fríos que los de la vertiente del Pacífico. La distribución de la temperatura media anual esta determinada por las variaciones altitudinales, los valores de más de $26^{\circ} \mathrm{C}$ se encuentran en la porción SO de la FVTM, y hasta menos de $2^{\circ} \mathrm{C}$ en las montañas más altas. Sin embargo, entre estos extremos, en la mayor parte de la zona de estudio la temperatura media anual se encuentra entre $12^{\circ}$ y $18^{\circ} \mathrm{C}$ siendo, sin embargo, más cálida la porción occidental, con valores entre $18^{\circ}$ y $22^{\circ} \mathrm{C}$. En toda la región, la primavera es la etapa más cálida y seca del año (Hernández-Cerda y Carrasco-Anaya, 2007). La vegetación montana que se desarrolla actualmente en la FVTM está constituida principalmente por bosques de Pinus (pinos), de Quercus (encinos) y de Pinus-Quercus que se establecen en altitudes de 1000 a $4000 \mathrm{msnm}$, por bosques mesófilos (1000 a 3200 msnm) y bosques de Abies (oyamel, 3000 a $3500 \mathrm{msnm}$ ). En las partes altas, por arriba de la línea arbolada $(4000$ msnm), se encuentra una vegetación de zacatonal alpino (Velázquez et al., 2000).

Las cuencas principales a lo largo de la FVTM en donde existen registros paleoambientales incluyen, de oriente a poniente, a las cuencas Oriental, de México, alto río Lerma, Cuitzeo, Zacapu, Pátzcuaro, y Zirahuén.

La cuenca Oriental $\left(4982 \mathrm{~km}^{2}\right)$ tiene una altitud media de unos $2300 \mathrm{msnm}$, con la presencia de once cráteres de explosión, seis de los cuales tienen actualmente un cuerpo de agua (axalapazcos). Estos seis lagos permanentes tienen agua entre levemente y marcadamente salobre, lo que refleja el clima relativamente seco de esta zona ( 425 a $656 \mathrm{~mm}$ de 
precipitación media anual), debido a la barrera orográfica de la Sierra Madre Oriental y de la cadena volcánica Cofre de Perote-Citlaltépetl.

La cuenca de México $\left(9600 \mathrm{~km}^{2}\right)$ albergó en el pasado un sistema lacustre con tres cuerpos principales, de norte a sur: Zumpango-Xaltocan, Texcoco, y Xochimilco-Chalco, además de algunos lagos menores en las planicies del noreste como Tecocomulco y Apan. Del sistema lacustre principal, el de menor altitud (aproximadamente $2215 \mathrm{msnm}$ ) era el lago de Texcoco, con aguas salobres, en tanto que los lagos de Zumpango-Xaltocan y Xochimilco-Chalco eran algunos metros más altos que el de Texcoco, y predominantemente dulces. Tecocomulco y Apan están hasta $250 \mathrm{~m}$ por arriba del sistema lacustre principal y en general están mucho menos impactados por la actividad humana que los lagos del sistema principal, los cuales han sido drenados y los ríos y manantiales que los alimentaban desviados y/o entubados. El clima en la cuenca tiene un gradiente de humedad, desde climas relativamente secos en el centro $(\sim 500 \mathrm{~mm} / \mathrm{año})$ y norte ( $\sim 600 \mathrm{~mm} / \mathrm{año})$, hacia climas más húmedos $(>800$ $\mathrm{mm} / \mathrm{año}$ ) en el sur, donde están las mayores elevaciones de la cuenca (volcanes Ajusco, Popocatépetl, e Iztaccíhuatl).

La cuenca alta del río Lerma yace al pie del Nevado de Toluca, a unos 2500 msnm, y tiene un clima más frío y húmedo (1000 mm/año) que las cuencas adyacentes. En ella hay tres lagos someros: Chignahuapan, Lerma y Chiconahuapan, los cuales han sido alterados por sobreexplotación y contaminación. Hacia la parte media de la cuenca del Lerma, existen diversas cuencas lacustres cerradas asociadas como Cuitzeo, Pátzcuaro, Zacapu y Zirahuén. Estas cuencas yacen en promedio a unos 2000 msnm y tienen climas más cálidos y húmedos que los de los sitios hacia el centro y este de la FVTM, recibiendo Cuitzeo las menores precipitaciones (700 mm/año) y Zirahuén las mayores (1160 mm/año).

\section{Marco cronológico}

La presente revisión se enfoca en los registros de avances glaciales, palinológicos y paleolimnológicos disponibles a lo largo de los volcanes y las cuencas de la FVTM para el UMG, pero considerará las fases previas y las posteriores con el propósito de establecer un marco de referencia. La cronología de la mayoría de los avances glaciares ha sido establecida mediante el isótopo cosmogénico ${ }^{36} \mathrm{Cl}$, reportándose sus edades en años antes del presente (años AP). Sin embargo, algunas secuencias glaciales y todas las paleolimnológicas y palinológicas para el centro de México han sido fechadas por el método del radiocarbono y sus modelos de edad han sido reportados en años de radiocarbono antes del presente $\left({ }^{14} \mathrm{C} \mathrm{AP}\right)$, que pueden ser calibrados a años calendáricos (Reimer et al., 2004; Weninger y Jöris, 2008). Las edades entre $10000{ }^{14} \mathrm{C}$ AP y $21000{ }^{14} \mathrm{C}$ AP fueron calibradas usando la base de datos INTCAL04 (Reimer et al. 2004), mientras que edades superiores (hasta $46000{ }^{14} \mathrm{C}$ AP inclusive) se calibraron con la base de datos de U/Th de Weninger y Jöris (2008). En ambos métodos de calibración para ${ }^{14} \mathrm{C}$ las edades se reportan como años antes del presente (AP), siendo el año 1950 el "presente". En este trabajo todos los datos presentados se expresan en años calendáricos antes del presente (cal AP), y divididos en cuatro ventanas de tiempo: Glacial Temprano (GTM), 25000-22000 cal AP (20.8-18.4 ${ }^{14} \mathrm{C}$ ka AP); Último Máximo Glacial (UMG), 22000-18000 cal AP (18.4-14.8 ${ }^{14} \mathrm{C}$ ka AP); Glacial Tardío (GTA), 1800015000 cal AP $\left(14.8-12.7{ }^{14} \mathrm{C}\right.$ ka AP) y Glacial Terminal (GTE), 15000-12000 cal AP (12.7-10 ${ }^{14} \mathrm{C}$ ka AP).

\section{Registros glaciales}

El registro de las glaciaciones en México se limita a las elevaciones de más de $3800 \mathrm{msnm}$, sólo existentes en la FVTM (Figura 1). Únicamente en las tres más altas -Pico de Orizaba o Citlaltépetl, Popocatépetl e Iztaccíhuatlsubsisten o subsistían hasta hace pocos años pequeños glaciares (Lorenzo, 1964).

Las evidencias de glaciación en estratovolcanes de la FVTM fueron estudiadas de manera sistemática en la segunda mitad del siglo XX por White (1962a,b, 1986), Heine (1975, 1988, 1994), White y Valastro (1984), y White et al. (1990). Recientemente se ha podido mejorar la cronología glacial del Iztaccíhuatl con fechamientos por ${ }^{36} \mathrm{Cl}$, así como extender los estudios a otras montañas (Vázquez-Selem, 2000; Vázquez-Selem y Heine, 2004).

El registro glacial más completo corresponde al Iztaccíhuatl, volcán de gran altura (5282 msnm) inactivo desde el Pleistoceno tardío, lo que favorece la preservación de las evidencias de glaciación. En el Popocatépetl (5452 msnm), el Pico de Orizaba (5675 msnm), el Nevado de Toluca (4560 msnm) y La Malinche (4461 msnm) el registro es fragmentario debido a la frecuente e intensa actividad volcánica ocurrida desde el Pleistoceno tardío. En el Popocatépetl el vulcanismo prácticamente ha borrado las huellas de glaciación, con excepción de las del Holoceno tardío; en las otras tres montañas se conserva un buen registro del Holoceno, pero el del Pleistoceno está en buena medida sepultado por lavas y depósitos piroclásticos. En cambio, en volcanes largamente inactivos como el Tancítaro (3842 msnm), el Cofre de Perote (4150 msnm) y el Ajusco (3952 msnm), se encuentran bien preservadas las huellas de glaciación, si bien se limitan al Pleistoceno tardío por ser montañas de menor altitud.

La altitud en la línea de equilibrio de los glaciares (ALE, también llamada línea de las nieves), y por consiguiente las fluctuaciones de los glaciares, están controladas fundamentalmente por la temperatura y secundariamente por la precipitación (Ohmura et al., 1992). En zonas de precipitación abundante la ALE desciende algo más que en zonas relativamente secas, aunque siempre dentro de los límites impuestos por la temperatura. La ALE coincide 
aproximadamente con la isoterma de $0{ }^{\circ} \mathrm{C}$. Por ello al reconstruir la ALE es posible estimar el descenso térmico en relación con la actualidad.

En la Tabla 1 se resumen las alturas mínimas de los glaciares y la ALE para varias montañas del centro de México y en la Tabla 2 se presenta la reconstrucción del descenso de temperatura basado en los datos del Iztaccíhuatl entre 21000 cal AP y el presente.

\subsection{Glacial temprano (GTM): 25000-22000 cal AP (ca. 21-18.4 ${ }^{14} \mathrm{C}$ ka AP)}

No hay registro de avances glaciales durante esta fase en ninguna de las montañas estudiadas en el centro de México. Evidencias de avances anteriores al GTM han sido identificadas en tres montañas, pero en los tres casos tienen edades mayores a los 25000 años: en el Iztaccíhuatl el avance glacial Nexcoalango (White 1962a,b; White et al., 1990), ha sido fechado en alrededor de 195000 años (Vázquez-Selem y Heine, 2004); en la Malinche hay depósitos glaciales de más de 32000 años (depósitos MI de Heine, 1988); y en el Ajusco de más de 25000 años (depósitos Marqués y Santo Tomás, de White y Valastro, 1984; White et al., 1990).

\section{2 Último Máximo Glacial (UMG): 22000-18000 cal AP (18.4-14.8 ${ }^{14} \mathrm{C}$ ka AP):}

Para esta fase hay registro de glaciación en todas las montañas de más de $3800 \mathrm{msnm}$. La evidencia está sustentada en fechamientos en seis montañas (Tancítaro, Nevado de Toluca, Ajusco, Iztaccíhuatl, Malinche, Cofre de Perote) y en correlaciones morfoestratigráficas en otras cuatro (Nevado de Colima, Telapón, Tláloc, Pico de Orizaba), si bien en estas últimas no se han hecho trabajos detallados. En el Iztaccíhuatl esta fase corresponde a lo que White (1962a,b) identificó como depósito glacial Hueyatlaco-1. White (1986) fechó este avance de manera tentativa entre $\sim 32000$ y 29500 cal AP $\left(\sim 27\right.$ y $25 \mathrm{ka}{ }^{14} \mathrm{C}$ AP). Más recientemente las morrenas Hueyatlaco-1 fueron fechadas mediante ${ }^{36} \mathrm{Cl}$, tefracronología y ${ }^{14} \mathrm{C}$ entre $\sim 21000$ y 17500 cal AP $\left(\sim 17.8-14.5 \mathrm{ka}{ }^{14} \mathrm{C} \mathrm{AP}\right.$; Vázquez-Selem, 2000), lo cual las sitúa claramente dentro del UMG. Los glaciares descendían hasta una altitud promedio de 3390 msnm y la ALE se encontraba alrededor de 3940 msnm (Tabla 1).

En el Tancítaro, volcán extinto de 3840 msnm situado en el centro-occidente de México, ocho fechamientos ${ }^{36} \mathrm{Cl}$ provenientes de tres valles del $\mathrm{W}$ y $\mathrm{N}$ señalan que su máximo avance glacial ocurrió antes de 18000 cal AP (14.8 $\left.\mathrm{ka}{ }^{14} \mathrm{C} \mathrm{AP}\right)$, y que éstos mantuvieron su máxima extensión hasta hace unos $15000 \mathrm{cal}$ AP $\left(12.7 \mathrm{ka}{ }^{14} \mathrm{C} \mathrm{AP}\right)$, alcanzando altitudes en torno a los $3200 \mathrm{msnm}$ y una ALE alrededor de los 3400 msnm (Lachniet y Vázquez-Selem, 2005). Las morrenas laterales fechadas se formaron presumiblemente durante el UMG, pero se mantuvieron activas después de 17000 cal AP (14.2 ka $\left.{ }^{14} \mathrm{C} \mathrm{AP}\right)$, por lo que probablemente representan una amalgama de los avances Hueyatlaco-1 y -2 del Iztaccíhuatl.

Algo similar se observa en el Cofre de Perote, donde nueve fechamientos ${ }^{36} \mathrm{Cl}$ en bloques morrénicos del máximo avance glacial sugieren que el desarrollo de las morrenas ocurrió de manera continua desde hace 20000 cal AP (18.4 $\mathrm{ka}{ }^{14} \mathrm{C}$ AP) hasta hace unos $14000 \mathrm{cal} \mathrm{AP}\left(12.1 \mathrm{ka}{ }^{14} \mathrm{C} \mathrm{AP}\right)$.

Tabla 1. Término y altitud de la línea de equilibrio (ALE) de los glaciares durante el Pleistoceno tardío en las montañas del centro de México.

\begin{tabular}{|c|c|c|c|c|c|c|c|c|c|c|}
\hline & \multicolumn{2}{|c|}{ Tancítaro } & \multicolumn{2}{|c|}{ Nevado de Toluca } & \multicolumn{2}{|c|}{ Ajusco } & \multicolumn{2}{|c|}{ Iztaccíhuatl } & \multicolumn{2}{|c|}{ Cofre de Perote } \\
\hline $\begin{array}{c}\text { Edad } \\
\text { (ka cal AP) }\end{array}$ & $\begin{array}{c}\text { Término } \\
\text { glaciares } \\
(\mathrm{msnm})\end{array}$ & $\begin{array}{c}\text { ALE } \\
(\mathrm{msnm}) \\
(1)\end{array}$ & $\begin{array}{c}\text { Término } \\
\text { glaciares } \\
(\mathrm{msnm})\end{array}$ & $\begin{array}{c}\text { ALE } \\
(\mathrm{msnm}) \\
(2)\end{array}$ & $\begin{array}{c}\text { Término } \\
\text { glaciares } \\
(\mathrm{msnm})\end{array}$ & $\begin{array}{c}\text { ALE } \\
(\mathrm{msnm}) \\
(3)\end{array}$ & $\begin{array}{c}\begin{array}{c}\text { Término } \\
\text { glaciares }\end{array} \\
(\mathrm{msnm})\end{array}$ & $\begin{array}{c}\text { ALE } \\
(\mathrm{msnm}) \\
(1)\end{array}$ & $\begin{array}{c}\text { Término } \\
\text { glaciares } \\
(\mathrm{msnm})\end{array}$ & $\begin{array}{c}\text { ALE } \\
(\mathrm{msnm}) \\
(1)\end{array}$ \\
\hline $\begin{array}{c}\text { Presente } \\
\text { (año 1960) }\end{array}$ & - & - & - & - & - & - & $4860 \pm 130$ & $4970 \pm 90$ & - & - \\
\hline $12-10$ & - & - & $\sim 3900$ & $\sim 4350$ & $?$ & $?$ & $3810 \pm 80$ & $4240 \pm 60$ & $3900-4000$ & $?$ \\
\hline $14-13$ & $3400 ?$ & $?$ & $?$ & $?$ & $?$ & $?$ & $\sim 3700$ & $\sim 4200$ & $3800 ?$ & $?$ \\
\hline $\begin{array}{l}17.5-14 \\
21-17.5\end{array}$ & $3150 \pm 70$ & $3390 \pm 50$ & $\sim 3400$ & $\sim 3800$ & $3150 ?$ & $3355 ?$ & $\begin{array}{l}3500 \pm 190 \\
3390 \pm 160\end{array}$ & $\begin{array}{l}4040 \pm 130 \\
3940 \pm 130\end{array}$ & $3390 \pm 70$ & $3650 \pm 40$ \\
\hline
\end{tabular}

(1) ALE calculada con el método Toe to Headwall Altitude Ratio (THAR), utilizando un cociente de 0.4.

(2) ALE estimada por altitud máxima de morrenas laterales.

(3) ALE calculada con el método Accumulation Area Ratio (AAR), utilizando un cociente de 0.65 (White et al., 1990).

Fuentes: Tancítaro, Lachniet y Vázquez-Selem (2005) e información no publicada de Vázquez-Selem; Ajusco (White et al., 1990); Nevado de Toluca, Heine (1994); Iztaccíhuatl, Vazquez-Selem (2000); Cofre de Perote, Lachniet y Vázquez-Selem (2005) e información no publicada de Vázquez-Selem. 
Tabla 2. Glaciares, ALE y estimaciones de temperaturas durante el Pleistoceno tardío en el Iztaccíhuatl.

\begin{tabular}{|c|c|c|c|c|c|c|c|}
\hline $\begin{array}{l}\text { Edad } \\
\text { (ka A.P.) }\end{array}$ & Fase & $\begin{array}{c}\text { Área } \\
\text { glaciada } \\
\left(\mathrm{km}^{2}\right)\end{array}$ & $\begin{array}{l}\text { Término } \\
\text { glaciares } \\
(\mathrm{msnm})\end{array}$ & $\begin{array}{c}\mathbf{A L E} \\
(\mathrm{msnm}) \\
(1)\end{array}$ & $\begin{array}{c}\text { Descenso de } \\
\text { ALE (m) } \\
(2)\end{array}$ & $\begin{array}{c}\text { Descenso térmico }\left({ }^{\circ} \mathrm{C}\right) \\
\text { GT: } 0.60^{\circ} \mathrm{C} / 100 \mathrm{~m} \\
(3)\end{array}$ & $\begin{array}{c}\text { Descenso térmico }\left({ }^{\circ} \mathrm{C}\right) \\
\text { GT: } 0.74^{\circ} \mathrm{C} / 100 \mathrm{~m} \\
(3)\end{array}$ \\
\hline 0.04 & Presente (1960) & 1.3 & $4860 \pm 130$ & $4970 \pm 90$ & 0 & 0 & 0 \\
\hline $12-10$ & Milpulco-1 & 46 & $3810 \pm 80$ & $4240 \pm 60$ & $730 \pm 60$ & $4.4 \pm 0.4$ & $5.4 \pm 0.5$ \\
\hline $17.5-14$ & Hueyatlaco-2 & 101 & $3500 \pm 190$ & $4040 \pm 130$ & $930 \pm 130$ & $5.6 \pm 0.8$ & $6.9 \pm 0.9$ \\
\hline $21-17.5$ & Hueyatlaco-1 & 131 & $3390 \pm 160$ & $3940 \pm 130$ & $1030 \pm 130$ & $6.2 \pm 0.8$ & $7.6 \pm 1.0$ \\
\hline
\end{tabular}

(1) ALE: altitud de la línea de equilibrio, calculada con el método Toe to Headwall Altitude Ratio (THAR), utilizando un cociente de 0.4.

(2) El descenso de la ELA se mide con respecto a la ELA del año 1960 (4970 msnm).

(3) El descenso térmico relativo a la actualidad (año 1960) se calcula utilizando dos diferentes gradientes térmicos (GT): 0.6 y $0.74{ }^{\circ} \mathrm{C} / 100 \mathrm{~m}$.

Fuente: Vázquez-Selem (2000).

Las morrenas indican que los glaciares descendían en promedio hasta la cota de $3390 \mathrm{msnm}$ y la ALE media se situaba en torno a la cota de $3650 \mathrm{msnm}$.

En el Nevado de Toluca morrenas similares a las del máximo avance del Pleistoceno en las tres montañas arriba mencionadas, indican que los glaciares en el lado norte llegaron cerca de los $3400 \mathrm{msnm}$ y permiten inferir una ALE alrededor de los $3800 \mathrm{msnm}$. Cabe mencionar que la actividad explosiva de fines del Pleistoceno hace difícil reconstruir la extensión de los glaciares y la ALE para esta fase, ya que buena parte de las laderas están sepultadas por decenas de metros de depósitos piroclásticos.

Algo similar ocurrió en La Malinche donde, sin embargo, Heine $(1975,1988)$ identificó huellas de un avance glacial (MII), fechado entre $\sim 18500$ y 14000 cal AP ( 15 a 12 ka ${ }^{14} \mathrm{C}$ AP) (Heine, 1994). Durante esta fase la línea climática de las nieves en el centro de México (ALE) se encontraba según Heine (1976) alrededor de 3800 msnm.

En resumen los registros apuntan a que durante esta fase en todos los grandes volcanes de la FVTM existían glaciares, y que la ALE estaba en su nivel más bajo, entre 3940 y 3400 msnm (es decir entre 1000 y 1500 m menos que hoy), con un menor descenso en los volcanes del centro (Iztaccíhuatl, Malinche y Nevado de Toluca) y un descenso más pronunciado en los más cercanos al mar (Cofre y Tancítaro).

\subsection{Glacial Tardío (GTA): 18000-15000 cal AP (14.8-12.7 ${ }^{14} \mathrm{C}$ ka AP)}

De esta fase datan las morrenas Hueyatlaco-2 del Iztaccíhuatl, originalmente descritas por White (1962a), quien les asignó una edad de $\sim 28000 \mathrm{cal} \mathrm{AP}\left(\sim 25 \mathrm{ka}{ }^{14} \mathrm{C} \mathrm{AP}\right)$ con base en correlaciones con el Ajusco (White, 1986). Más recientemente, fueron fechadas por ${ }^{36} \mathrm{Cl}$, tefracronología y ${ }^{14} \mathrm{C}$ entre más de 17500 y $14000 \mathrm{cal} \mathrm{AP}\left(<14.5\right.$ y $12.1 \mathrm{ka}{ }^{14} \mathrm{C}$ AP; Vázquez-Selem y Heine, 2004); es decir son posteriores al UMG. Estas morrenas, masivas y muy próximas a las del avance anterior, indican que entre $\sim 17500$ y 14000 cal AP el término de los glaciares (3500 msnm) y la ALE (4040 $\mathrm{msnm}$ ) se encontraba apenas $\sim 100 \mathrm{~m}$ por encima de los del UMG.

En el Tancítaro, el Cofre de Perote y Malinche no se puede distinguir claramente dos fases separadas de glaciación equivalentes a Hueyatlaco-1 y -2 del Iztaccíhuatl. Como se señala arriba, la cartografía y los fechamientos sugieren que los glaciares se mantuvieron con dimensiones muy similares desde el UMG (22000-18000 cal AP) hasta hace $\sim 14000$ cal AP, tal vez adelgazándose gradualmente pero sin retirarse de sus posiciones máximas. Como se señaló, el avance MII de La Malinche se extiende hasta hace unos $14000 \mathrm{cal}$ AP (12 ka ${ }^{14} \mathrm{C} \mathrm{AP}$; Heine, 1994), por lo cual cae dentro del GTA, si bien la actividad explosiva al final y después de esta fase (Castro-Govea y Siebe, 2007) impiden una reconstrucción detallada de los glaciares. Lo mismo se aplica al Nevado de Toluca, caracterizado por una intensa actividad explosiva entre 15500 y $14000 \mathrm{cal}$ AP (Arce et al., 2005; D'Antonio et al., 2008).

En el Ajusco, White y Valastro (1984), White (1986) y White et al. (1990) identificaron un avance durante el cual dos glaciares de valle descendieron hasta $\sim 3200 \mathrm{msnm}$, con una ALE a $3555 \mathrm{~m} \mathrm{snm}$, situándolo de manera tentativa entre 18000 y $11500 \mathrm{cal} \mathrm{AP}\left(15-10 \mathrm{ka}{ }^{14} \mathrm{C} \mathrm{AP}\right)$, por lo cual parece corresponder a esta fase glacial.

En resumen durante esta fase todos los volcanes continúan con glaciares, en algunos (Cofre, Tancítaro) todavía en su máxima extensión, mientras en el Iztaccíhuatl se nota un pequeño ascenso de la ALE de $\sim 100 \mathrm{~m}$.

\subsection{Glacial Terminal (GTE): 15000-12000 cal AP (12.7- $10{ }^{14} \mathrm{C}$ ka AP)}

Hace 15000 años, al inicio de este periodo, los glaciares se encontraban aún en el máximo del avance Hueyatlaco-2 
del Iztaccíhuatl (y equivalentes en el Tancítaro y Cofre de Perote). Sin embargo, fechamientos ${ }^{36} \mathrm{Cl}$ en varios valles indican que entre 15000 y $14000 \mathrm{cal}$ AP $(12.7-12.1 \mathrm{ka}$ ${ }^{14} \mathrm{C} \mathrm{AP}$ ) los glaciares retrocedieron, primero lentamente y en pulsos, deteniéndose momentáneamente para formar pequeñas morrenas recesionales. Sin embargo, después de 14000 cal AP el retroceso ocurrió rápidamente y se prolongó hasta poco antes de $12500 \mathrm{cal}$ AP. La mayoría de las laderas y valles del Iztaccíhuatl por debajo de 4000-3900 msnm se encontraban sin glaciares hace unos $12500 \mathrm{cal}$ AP (10.5 ka ${ }^{14} \mathrm{C}$ AP). Los datos disponibles para montañas de altitud media como el Tancítaro y el Cofre de Perote indican una deglaciación casi total (con la posible excepción de los circos cercanos a las cimas) hace unos 12000 años.

Sin embargo, en el Iztaccíhuatl los glaciares volvieron a avanzar (o bien dejaron de retroceder y se estabilizaron durante algún tiempo) llegando hasta $\sim 3800-3900 \mathrm{msnm}$ en los valles principales, formando las morrenas Milpulco-1 (White, 1962a, 1986), con una ALE promedio de 4250 $\mathrm{msnm}$. Este avance culminó hace $\sim 12500 \mathrm{cal}$ AP y se registró al menos en el Iztaccíhuatl y en el Nevado de Toluca (Vázquez-Selem y Heine, 2004). Las pequeñas morrenas frontales sugieren que el máximo fue de corta duración (tal vez menos de 500 años) y fue seguido de una fase de retroceso lento, en pequeños pulsos, que se prolongó hasta hace $\sim 10500 \mathrm{cal}$ AP $\left(9.3 \mathrm{ka}{ }^{14} \mathrm{CAP}\right)$ cuando los fondos de valle a 3800-4000 msnm quedaron descubiertos de hielo. En la Malinche, las morrenas MIII de Heine $(1988,1994)$ aparentemente corresponden a esta fase.

\section{Registros lacustres}

Existen pocos registros lacustres en el centro de México que abarquen los últimos 25000 años; los registros disponibles se muestran en la Figura 1 y se resumen en la Tabla 3. Los sitios se distribuyen de manera general en tres regiones geográficas. En la porción oriente de la FVTM el único registro disponible que abarca los últimos $25000 \mathrm{cal}$ AP es el Jalapasquillo (Ohngemach y Straka, 1983; Straka y Ohngemach, 1989), localizado en la cuenca Oriental (2400 $\mathrm{msnm}$ ). Existe un registro de la cuenca Puebla-Tlaxcala, que proviene del cráter del volcán La Malinche (Tláloc II). La edad de esta secuencia es desconocida, dado que sólo cuenta con un fechamiento radiométrico en la cima (7.9 ka ${ }^{14} \mathrm{C} \mathrm{AP}$ ) pero los autores consideran que se extiende hasta el Pleistoceno tardío. En la parte central de la FVTM se localiza la cuenca de México, donde existen varios registros que cubren los últimos $25000 \mathrm{cal} \mathrm{AP}$; de norte a sur los sitios son: Tecocomulco, Texcoco y Chalco. En la porción central de la FVTM también se ubica la cuenca alta del Lerma, en donde se tienen los registros de Chignahuapan y Metepec. En la porción centro-oeste de la FVTM las cuencas con un registro que abarca los últimos 25000 años incluyen a Cuitzeo, Pátzcuaro y Zacapu; Zirahuén tiene un registro que sólo abarca los últimos 17000 cal AP.

\subsection{Glacial Temprano (GTM): 25000-22000 cal AP (ca.} $21-18.4{ }^{14} \mathrm{C}$ ka AP)

En el diagrama polínico del Jalapasquillo, cuenca Oriental (Tabla 3), el principal componente del registro es Pinus (pino, 90\%) y en menor proporción Quercus (encino, 10\%), además de la presencia constante, aunque con bajos valores bajos $(<5 \%)$, de Picea, lo que sugiere que el área estaba cubierta con bosques de coníferas, principalmente de Pinus, con un ambiente más frío y húmedo que el actual (Straka y Ohngemach, 1989). Este registro carece de otro tipo de datos que permitan reconstruir las condiciones limnológicas del sitio y en general su control cronológico es pobre.

Para Tecocomulco los núcleos estudiados (Tabla 3) corresponden a un sitio cercano a la orilla del lago moderno. En estas secuencias, el registro de polen y diatomeas es pobre durante el UMG. Las diatomeas están ausentes a partir de los $c a .31000 \mathrm{cal} \mathrm{AP}\left(26 \mathrm{ka}{ }^{14} \mathrm{C} \mathrm{AP}\right)$ y la presencia de una capa rica en carbonatos hacia los 30000 cal AP (25 ka ${ }^{14} \mathrm{C} \mathrm{AP}$ ) indica el establecimiento de condiciones más secas para el inicio del GTM (Roy et al., 2009), pasando de condiciones propiamente lacustres antes de $30000 \mathrm{cal}$ AP hacia condiciones pantanosas o subaéreas durante el GTM. La única muestra de polen que corresponde al GTM sugiere la presencia de bosques de Pinus y un ambiente de pantano con Isoëtes, un helecho subacuático que se desarrolla en climas de templados a semi-fríos. Esto es, en condiciones más frías que las actuales en esta localidad (Caballero et al., 1999).

Para el lago de Texcoco hay varias secuencias analizadas desde la zona litoral hacia la zona central del lago. El registro lacustre-edáfico de Tepexpan, localizado en la orilla NE del lago, ha producido interpretaciones diversas (SolleiroRebolledo et al., 2006; Lamb et al., 2009; Sedov et al., $2009 b$ ). Se puede concluir, sin embargo, que el ambiente lacustre dominante entre 25000 y quizás hasta $18000 \mathrm{cal}$ AP era somero y salobre; según Sedov et al. (2009b) los datos polínicos indican que bosques de Pinus y Quercus se desarrollaban cercanos al sitio de Tepexpan durante el GTM. Existen otras secuencias hacia la zona central del lago (Tabla 3) en las que hay una presencia constante de diatomeas indicadoras de condiciones someras y salobres, posiblemente con un leve incremento en la salinidad a partir de 28000 cal AP $\left(23 \mathrm{ka}{ }^{14} \mathrm{C}\right.$ AP; Bradbury, 1971, 1989; Ramírez-Nava, 2002). También la presencia de taxa de pantano como Isoëtes indica niveles lacustres bajos (Lozano-García y Ortega-Guerrero, 1998; SandovalMontaño, 2000). En los diagramas polínicos hay evidencias de bosques de Pinus, con escasa presencia de Quercus y del tipo Cupressaceae, que se asocia a condiciones secas, mientras que las herbáceas no son abundantes. Hay también registros de Picea, una conífera no presente en la actualidad en la zona central de México, que se desarrolla en ambientes fríos; están presentes otros elementos arbóreos mesófilos 
Tabla 3. Inventario de sitios con registros palinológicos y/o paleolimnológicos entre 25000 a 12000 cal AP a lo largo de la FVTM.

\begin{tabular}{|c|c|c|c|c|c|c|c|c|c|c|c|c|}
\hline Sitio & $\begin{array}{l}\text { Longitud- } \\
\text { Latitud }\end{array}$ & $\begin{array}{r}\text { Altitud } \\
(\mathrm{msnm})\end{array}$ & $\begin{array}{l}\text { Precip. } \\
(\mathrm{mm} / \mathrm{año})\end{array}$ & $\begin{array}{l}\text { Temp. } \\
\left({ }^{\circ} \mathrm{C}\right)\end{array}$ & Registro & Posición & $\begin{array}{l}\text { Loquitud } \\
\text { (m) }\end{array}$ & Fechas & $\begin{array}{l}\text { Edad máx. } \\
\left({ }^{14} \mathrm{C} \text { kaBP }\right)\end{array}$ & $\begin{array}{l}\text { Edad máx. } \\
\text { (cal KaBP) }\end{array}$ & $\begin{array}{l}\text { Análisis } \\
\text { realizados }\end{array}$ & Referencia \\
\hline Oriental & $\begin{array}{l}97^{\circ} 25^{\prime} \mathrm{W} \\
19^{\circ} 13^{\prime} \mathrm{N}\end{array}$ & 2400 & 400 & 14.4 & $\begin{array}{c}\text { Jalapasquill } \\
\text { o }\end{array}$ & $?$ & 20 & $3\left({ }^{14} \mathrm{C}\right)$ & 35 & 41 & $\mathrm{P}$ & $\begin{array}{l}\text { Ohngemach y Straka, 1983; Straka y } \\
\text { Ohngemach, } 1989\end{array}$ \\
\hline Malinche & $\begin{array}{l}98^{\circ} 02^{\prime} \mathrm{W} \\
19^{\circ} 13^{\prime} \mathrm{N}\end{array}$ & 3100 & 650 & 14 & Tlaloc II & ? & 3 & $1\left({ }^{14} \mathrm{C}\right)$ & $?$ & $?$ & $\mathrm{P}$ & $\begin{array}{l}\text { Ohngemach y Straka, 1983; Straka y } \\
\text { Ohngemach, } 1989\end{array}$ \\
\hline \multirow[t]{3}{*}{ Tecocomulco } & \multirow{3}{*}{$\begin{array}{l}98^{\circ} 23^{\prime} \mathrm{W} \\
19^{\circ} 51^{\prime} \mathrm{N}\end{array}$} & \multirow[t]{3}{*}{2500} & \multirow[t]{3}{*}{600} & \multirow[t]{3}{*}{15} & T-A, T-B & orilla S & 6 & $5\left({ }^{14} \mathrm{C}\right)$ & $>45$ & $>46.5$ & $\begin{array}{l}\text { MS, MM, CO, } \\
\text { P, D, GQ }\end{array}$ & Caballero et al., 1999; Roy et al., 2009 \\
\hline & & & & & T-A95 & orilla S & 2.55 & $1(\mathrm{~T})$ & 31 & 33 & $\mathrm{GQ}$ & Roy et al., 2009 \\
\hline & & & & & CHB (CHA) & $\begin{array}{l}\text { centro } \\
\text { pantano }\end{array}$ & 26 & $\begin{array}{l}7\left({ }^{14} \mathrm{C}\right) \\
2(\mathrm{~T})\end{array}$ & 47 & $>48$ & MS, CO, D, P & $\begin{array}{l}\text { Lozano et al., 1993; Caballero y Ortega, } \\
1998\end{array}$ \\
\hline \multirow[t]{3}{*}{ Chalco } & \multirow[t]{3}{*}{$\begin{array}{l}98^{\circ} 02^{\prime} \mathrm{W} \\
19^{\circ} 13^{\prime} \mathrm{N}\end{array}$} & \multirow[t]{3}{*}{2240} & \multirow[t]{3}{*}{680} & \multirow[t]{3}{*}{15} & CHD & $\begin{array}{l}\text { centro } \\
\text { pantano }\end{array}$ & 12 & $\begin{array}{l}8\left({ }^{14} \mathrm{C}\right) \\
2(\mathrm{~T})\end{array}$ & 24 & 29 & MS, P & Lozano y Ortega-Guerrero, 1994 \\
\hline & & & & & CHE & $\begin{array}{l}\text { centro } \\
\text { pantano }\end{array}$ & 10 & $\begin{array}{l}14\left({ }^{14} \mathrm{C}\right) \\
6(\mathrm{~T})\end{array}$ & 16 & 18 & MS, P & Sosa y Nájera, 2001 \\
\hline & & & & & TXB & $\begin{array}{l}\text { centro } \\
\text { seco }\end{array}$ & 10.8 & $\begin{array}{l}5\left({ }^{14} \mathrm{C}\right) \\
2(\mathrm{~T})\end{array}$ & 34 & 40 & MS, MM, D, P & $\begin{array}{l}\text { Lozano y Ortega-Guerrero, 1998, } \\
\text { Ramírez, } 2002\end{array}$ \\
\hline \multirow{4}{*}{ Texcoco } & \multirow{4}{*}{$\begin{array}{l}98^{\circ} 00^{\prime} \mathrm{W} \\
19^{\circ} 29^{\prime} \mathrm{N}\end{array}$} & \multirow{4}{*}{2216} & \multirow{4}{*}{550} & \multirow{4}{*}{15.6} & TXA & $\begin{array}{l}\text { centro } \\
\text { seco }\end{array}$ & 6.5 & $\begin{array}{l}4\left({ }^{14} \mathrm{C}\right) \\
3(\mathrm{~T})\end{array}$ & $>27$ & $>35$ & MS, P & Sandoval-Montaño, 2000 \\
\hline & & & & & Cruz de Lago & $\begin{array}{l}\text { centro } \\
\text { seco }\end{array}$ & 7.2 & $\begin{array}{l}3\left({ }^{14} \mathrm{C}\right) \\
2(\mathrm{~T})\end{array}$ & 35 & 41 & $\mathrm{D}, \mathrm{P}$ & $\begin{array}{l}\text { González-Quintero y Fuentes-Mata, 1980; } \\
\text { Bradbury, 1971, } 1989\end{array}$ \\
\hline & & & & & Tepexpan & orilla NE & 4.5 & $4\left({ }^{14} \mathrm{C}\right)$ & 28 & 32 & GQ, P, I & Sedov et al ., 2009 \\
\hline & & & & & Tepexpan & orilla NE & 4 & $5\left({ }^{14} \mathrm{C}\right)$ & 19 & 23 & $\mathrm{CO}, \mathrm{GQ}, \mathrm{D}, \mathrm{I}$ & Lamb et al., 2009 \\
\hline \multicolumn{13}{|l|}{ Alto Lerma } \\
\hline \multirow{3}{*}{ Chignahuapan } & \multirow{3}{*}{$\begin{array}{l}99^{\circ} 32^{\prime} \mathrm{W} \\
19^{\circ} 10^{\prime} \mathrm{N}\end{array}$} & \multirow{3}{*}{2570} & \multirow{3}{*}{1000} & \multirow{3}{*}{12.2} & Sta. Cruz & $\begin{array}{l}\text { litoral E, } \\
\text { seco }\end{array}$ & 9.54 & $\begin{array}{l}6\left({ }^{14} \mathrm{C}\right) \\
2(\mathrm{~T})\end{array}$ & 22 & 27 & $\begin{array}{l}\text { MS, MM, CO, } \\
\text { CA, P, D }\end{array}$ & Caballero et al., 2002 \\
\hline & & & & & Almoloya & E, seco & 5.1 & $\begin{array}{l}4\left({ }^{14} \mathrm{C}\right) \\
2(\mathrm{~T})\end{array}$ & 12.5 & 14.6 & $\mathrm{D}$ & Caballero et al., 2002 \\
\hline & & & & & Isla II & $\begin{array}{l}\text { litoral } \mathrm{W} \\
\text { seco }\end{array}$ & 5.7 & $\begin{array}{l}2\left({ }^{14} \mathrm{C}\right) \\
3(\mathrm{~T})\end{array}$ & 14 & 16.7 & MS, CO, D & Caballero et al., 2001 \\
\hline Metepec & $\begin{array}{l}99^{\circ} 35^{\prime} \mathrm{W} \\
19^{\circ} 14^{\prime} \mathrm{N}\end{array}$ & 2600 & 850 & 13 & Metepec & orilla W & 7 & $\begin{array}{l}4\left({ }^{14} \mathrm{C}\right) \\
4(\mathrm{~T})\end{array}$ & 37 & 41.8 & MS, P & Caballero et al., 2001 \\
\hline Cuitzeo & $\begin{array}{l}101^{\circ} 08^{\prime} \mathrm{W} \\
19^{\circ} 55^{\prime} \mathrm{N}\end{array}$ & 1880 & 700 & 17 & $\begin{array}{c}\text { Pozo } \\
\text { Cuitzeo }\end{array}$ & $\begin{array}{l}\text { centro } \\
\text { seco }\end{array}$ & 27 & $10\left({ }^{14} \mathrm{C}\right)$ & $>100(?)$ & & D & Israde et al ., 2002 \\
\hline \multirow[t]{2}{*}{ Patzcuaro } & \multirow[t]{2}{*}{$\begin{array}{l}101^{\circ} 39^{\prime} \mathrm{W} \\
19^{\circ} 36^{\prime} \mathrm{N}\end{array}$} & \multirow[t]{2}{*}{2000} & \multirow[t]{2}{*}{950} & \multirow[t]{2}{*}{16.3} & 1973 Core & $\begin{array}{l}\text { S, bajo } 6.2 \\
\mathrm{~m}\end{array}$ & $15.2 / 6.2$ & $7\left({ }^{14} \mathrm{C}\right)$ & 44 & 45.5 & GQ, D, P & Watts y Bradbury, 1982; Bradbury, 2000 \\
\hline & & & & & $\mathrm{C} 4$ & $\begin{array}{l}\mathrm{N} \text {, bajo } 2.5 \\
\mathrm{~m}\end{array}$ & $7.35 / 6.2$ & $11\left({ }^{14} \mathrm{C}\right)$ & 19 & 22.5 & $\mathrm{CO}, \mathrm{GQ}, \mathrm{D}$ & Metcalfe et al., 2007 \\
\hline Zirahuen & $\begin{array}{l}101^{\circ} 44^{\prime} \mathrm{W} \\
19^{\circ} 26^{\prime} \mathrm{N}\end{array}$ & 2200 & 1160 & 16.2 & ZIR03-I & $\begin{array}{l}\text { litoral, } \\
\text { bajo } 12 \mathrm{~m}\end{array}$ & $6.61 / 12$ & $10\left({ }^{14} \mathrm{C}\right)$ & 14 & 17 & $\begin{array}{l}\text { M, MM, CO, } \\
\text { GQ, P, D }\end{array}$ & Ortega et al ., 2010 \\
\hline \multirow{3}{*}{ Zacapu } & \multirow{3}{*}{$\begin{array}{l}101^{\circ} 40^{\prime} \mathrm{W}, \\
19^{\circ} 50^{\prime} \mathrm{N}\end{array}$} & \multirow{3}{*}{2000} & \multirow{3}{*}{850} & 17 & Cantabria I & $\begin{array}{l}\text { centro } \\
\text { seco }\end{array}$ & 11 & $9\left({ }^{14} \mathrm{C}\right)$ & 52 & ? & MS, MM, CO & Ortega et al., 2002 \\
\hline & & & & & Ladrillera & orilla & 5 & $3\left({ }^{14} \mathrm{C}\right)$ & 30 & 34.2 & D & Metcalfe, 1992 \\
\hline & & & & & Sección 1 & orilla & 1.7 & $1\left({ }^{14} \mathrm{C}\right)$ & ? & $?$ & D & Metcalfe, 1992 \\
\hline
\end{tabular}

MS = susceptibilidad magnética, $\mathrm{MM}=$ mineralogía magnética, $\mathrm{CO}=$ carbón orgánico, $\mathrm{CA}=$ partículas de carbón, $\mathrm{GQ}=\mathrm{geoquímica}, \mathrm{I}=$ isótopos estables, $\mathrm{P}=$ polen y palinomorfos, $\mathrm{D}=$ diatomeas.

como Podocarpus y Abies (oyamel). Probablemente estos taxa se desarrollaban en ambientes localmente más húmedos, como cañadas (Lozano-García y Ortega-Guerrero, 1998; Sandoval-Montaño, 2000).

Las secuencias estudiadas en Chalco corresponden a la zona central del lago. En este sitio también se detecta una tendencia hacia una reducción en el nivel lacustre con condiciones someras y salobres desde los 31000 cal AP $\left(27.5 \mathrm{ka}{ }^{14} \mathrm{C} \mathrm{AP}\right)$, pero en Chalco el GTM está marcado por un cambio brusco de condiciones salobres a condiciones de agua dulce a los $26500 \mathrm{cal} \mathrm{AP}\left(22 \mathrm{ka}{ }^{14} \mathrm{C} \mathrm{AP}\right)$ que sugieren un incremento en el aporte de agua al lago. Esto pudo estar asociado con un cambio hidrológico local de origen volcánico (Caballero y Ortega, 1998), ya que es el único registro en la cuenca de México que marca un incremento de nivel lacustre durante este tiempo. Los registros de los espectros polínicos del lago de Chalco marcan también un cambio en la composición de los conjuntos de polen, con la disminución significativa de Pinus y aumento en Cupressaceae y pastos; esta señal polínica de la vegetación continúa hasta el UMG y sugiere condiciones menos húmedas (Lozano-García et al., 1993; Lozano-García y Ortega-Guerrero, 1994; Lozano-García, 1996).

El registro de la cuenca alta del Lerma incluye la secuencia litoral de Metepec (Caballero et al., 2001), de la que se rescataron restos de un mamut en niveles fechados en $\sim 31000$ cal AP (28 a 24 ka ${ }^{14} \mathrm{C}$ AP). Durante el GTM se presenta un cambio de un ambiente palustre hacia un ambiente fluvial, en el que domina Isoëtes, indicando ambientes someros y fríos; los conjuntos herbáceos dominan sobre los arbóreos, indicando bosques muy abiertos. Hacia el este de la planicie lacustre de Chignahuapan el registro 
inicia a los $26500 \mathrm{cal} \mathrm{AP}\left(22 \mathrm{ka}{ }^{14} \mathrm{CAP}\right.$, Tabla 3$)$. Éste indica que durante el GTM hay un bajo aporte de sedimentos hacia el lago. El lago es de agua dulce con algunas fluctuaciones, levemente más profundo hacia los $24000 \mathrm{cal} \mathrm{AP}\left(20 \mathrm{ka}^{14} \mathrm{C}\right.$ AP) indicado por la presencia de especies de diatomeas planctónicas y más somero hacia los 22500 cal AP (19 ka ${ }^{14} \mathrm{C} \mathrm{AP}$ ) sugerido por la presencia de hematita, que indica exposición subaérea. En el diagrama palinológico se observa un aumento de los conjuntos herbáceos y la reducción de los bosques cerca de los $26000 \mathrm{cal} \mathrm{AP}\left(21.3 \mathrm{ka}{ }^{14} \mathrm{C} \mathrm{AP}\right)$, lo cual se ha interpretado como el desarrollo de bosque abierto como resultado de los desplazamientos de la franja de vegetación en respuesta a las condiciones frías (Caballero et al., 2002; Lozano-García et al., 2005).

En Cuitzeo se cuenta con un núcleo en la parte central del lago. En esta secuencia, una intensa actividad volcánica interrumpió el registro lacustre hacia los $30000 \mathrm{cal}$ AP (25 ka ${ }^{14} \mathrm{C} \mathrm{AP}$ ), lo que limita la preservación de diatomeas y polen. Los datos indican la presencia de un lago somero levemente salobre hacia los $24000 \mathrm{cal} \mathrm{AP}\left(20 \mathrm{ka}{ }^{14} \mathrm{C} \mathrm{AP}\right)$. A los $22000 \mathrm{cal} \mathrm{AP}\left(18.4 \mathrm{ka}{ }^{14} \mathrm{C} \mathrm{AP}\right)$ se registran valores muy altos de la diatomea Stephanodisccus niagarae que se han interpretado como indicadora de un evento corto de mayor nivel lacustre. En los conjuntos polínicos hay presencia de polen de herbáceas como pastos y compuestas y en menor proporción están presentes elementos arbóreos, indicando posiblemente la perturbación por la actividad volcánica (Velázquez-Durán, 2003).

En Pátzcuaro, el registro que abarca los últimos 25000 cal AP es un núcleo perforado hacia la parte sur del lago en 1973 (Tabla 2; Watts y Bradbury, 1982; Bradbury, 2000). Con base en los datos de diatomeas de este núcleo, Bradbury (2000) considera que durante el GTM el lago era profundo y turbio por un mayor aporte de sedimentos a la cuenca y que dominaba un patrón de lluvias en invierno, con verano cálido. Con respecto a los datos de vegetación los taxa dominantes son Pinus, Quercus, y Alnus (aile), éstos presentan fluctuaciones en sus valores aunque el elemento dominante es el Pinus.

En Zacapu se tiene una tasa de sedimentación muy baja para este período y no hay evidencias que permitan suponer la presencia de un cuerpo de agua entre 30000 y $18500 \mathrm{cal}$ AP (25 y 15 ka ${ }^{14} \mathrm{C} \mathrm{AP}$; Metcalfe, 1992; Ortega et al., 2002). En cuanto al registro de polen, entre 30000 y $22500 \mathrm{cal}$ AP (25 a $19 \mathrm{ka}^{14} \mathrm{C}$ AP) está dominado solamente por Pinus, con la ausencia casi total de Quercus y Alnus (Lozano-García y Sosa-Nájera, datos inéditos).

\section{2 Último Máximo Glacial (UMG): 22000-18000 cal AP (18.4-14.8 ${ }^{14} \mathrm{C}$ ka AP)}

En el diagrama polínico del Jalapasquillo destaca la abundancia de polen de herbáceas a $\sim 21000$ cal AP (18 ka ${ }^{14} \mathrm{C} \mathrm{AP}$ ), con algunos elementos mesofíticos, y también se registra Picea, abundante Pinus y en menor proporción Quercus. Aunque la cronología del sitio es limitada (3 fechas en $20 \mathrm{~m}$ de registro), la interpretación paleoclimática que realizan Straka y Ohngemach (1989) para este periodo es de una transición de climas fríos y secos a climas fríos y húmedos.

En la cuenca de México, las secuencias del norte (Tecocomulco y Texcoco) aportan evidencias de una reducción en la humedad disponible con respecto al GTM. En Tecocomulco a partir de los ca. $19000 \mathrm{cal}$ AP (16 ka 14C AP) se establecen condiciones muy secas durante las cuales no hay evidencias de un ambiente lacustre, se registra una tasa de sedimentación muy baja sin preservación de diatomeas ni de polen; los datos geoquímicos indican un intemperismo bajo asociado a condiciones secas. Durante esta etapa se depositaron varias capas sucesivas de caliche que probablemente datan de hace 19000 a $18500 \mathrm{cal}$ AP (16-15 ka ${ }^{14} \mathrm{C} \mathrm{AP}$ ) y que indican condiciones alternantes de inundación y exposición en el sitio (Caballero et al., 1999; Roy et al., 2009).

En Texcoco, en la secuencia litoral de Tepexpan, a pesar de las discrepancias cronológicas mencionadas, se infiere la presencia de un lago somero, salobre, con la presencia de Ruppia maritima (Sedov et al., 2009b). Estos autores infieren para este tiempo la presencia de bosques de Pinus y Quercus que pasan a ser dominados sólo por Pinus. En los registros del centro del lago esta etapa tiene la mayor diversidad de diatomeas, sugiriendo ambientes someros, pero levemente menos salobres (Ramírez-Nava, 2002); al mismo tiempo hay un aumento de ciperáceas, lo que indica que la zona central pasó a ser un ambiente litoral. Es posible que la influencia de la entrada de agua dulce de los ríos y/o manantiales haya favorecido un ambiente localmente menos salobre, como lo indican las diatomeas. Para los diagramas palinológicos del centro de lago TX-A y TX-B antes de $21300 \mathrm{cal} \mathrm{AP}\left(18 \mathrm{ka}{ }^{14} \mathrm{C} \mathrm{AP}\right)$ se reporta en la vegetación regional la presencia de bosques de Pinus y Quercus; Picea está presente, aunque con valores bajos. Se registra además el incremento de polen de elementos herbáceos que caracteriza a los conjuntos polínicos durante el UMG. En general se infieren condiciones menos húmedas con base en la disminución del nivel lacustre y el aumento de los elementos herbáceos.

En Chalco el lago experimenta un cambio importante a los 22000 cal AP ( $\left.c a .18 .5 \mathrm{ka}{ }^{14} \mathrm{C} \mathrm{AP}\right)$, con un aumento en las ciperáceas y un conjunto de diatomeas (Cocconeis y Eunotia) que indica una reducción en el nivel lacustre; este ambiente somero es muy variable y alterna entre condiciones de pantano ácido a neutral. El impacto de la actividad volcánica es muy intenso y es probable que haya afectado al lago. Los valores de concentración de granos de polen en las secuencias son bajos y la composición de los conjuntos de polen indica la existencia de bosques de Pinus y Pinus-Quercus con abundantes pastos, lo que sugiere bosques abiertos. Picea está presente $(5 \%)$ en el núcleo CHA-E indicando que las condiciones prevalecientes eran muy frías en el sur de la cuenca de México. La presencia abundante de polen de Mimosa aff. biuncifera (70-80\%) 
en los diagramas a los 20000 y $18500 \mathrm{ka} \mathrm{AP} \mathrm{(17} \mathrm{y} 15 \mathrm{ka}$ ${ }^{14} \mathrm{C} \mathrm{AP}$ ) sugiere que condiciones secas se extendieron hasta la porción sur de la cuenca durante el UMG, siendo muy probablemente el periodo más seco de los últimos $25000 \mathrm{cal}$ AP (Lozano-García et al., 1993; Lozano-García y OrtegaGuerrero, 1994; Lozano-García, 1996; Sosa-Nájera, 2001).

En la cuenca alta del río Lerma (Chignahuapan) se registra un incremento en el aporte de sedimentos al lago y una leve reducción en el nivel lacustre con un aumento en la vegetación subacuática entre 21300 y 19100 cal AP (18 y $16 \mathrm{ka}{ }^{14} \mathrm{C}$ AP). Después de los $19100 \mathrm{cal}$ AP $\left(16 \mathrm{ka}{ }^{14} \mathrm{C}\right.$ AP) el lago se recupera y la erosión disminuye. Los bosques son muy abiertos, el diagrama de polen presenta un máximo de elementos herbáceos (pastos) a los 20800 y $19100 \mathrm{cal}$ AP (17.5 y $\left.16 \mathrm{ka}{ }^{14} \mathrm{C} \mathrm{AP}\right)$. Siendo la cuenca más altas del transecto analizado, es probable que los conjuntos polínicos correspondientes al periodo más frío estén compuestos por elementos de la vegetación alpina que son básicamente zacatonales, los cuales descendieron durante los avances glaciales (Caballero et al., 2002; Lozano-García et al., 2005).

En la parte occidental de la FVTM en el lago de Cuitzeo, entre $\sim 22000$ y 21000 cal AP (ca. 18.5 a 17.6 ka ${ }^{14} \mathrm{CAP}$ ) está presente un lago somero y salobre. En el registro polínico se identifican Pinus, Quercus y Alnus; sin embargo sus valores son bajos, mientras que los conjuntos de herbáceas (pastos, compuestas y Chenopodiaceas) son abundantes, llegando a valores máximos de $80 \%$. En la estratigrafía del núcleo de Cuitzeo se observa una sedimentación sumamente baja entre 21000 y 10000 cal AP (17.6 a 8.8 ka $\left.{ }^{14} \mathrm{C} \mathrm{AP}\right)$, que posiblemente refleja un hiato en la secuencia asociado con ambientes lacustres muy someros indicativos de condiciones secas.

En Pátzcuaro, en los dos registros que cubren esta ventana de tiempo (Bradbury, 2000; Metcalfe et al., 2007) las diatomeas indican un lago profundo y frío; de acuerdo con Bradbury (2000) este lago continúa bajo un régimen de inviernos fríos y húmedos, durante los cuales posiblemente se congelaba, y con veranos relativamente secos, pero probablemente con una mayor variabilidad en el ciclo anual. Los datos palinológicos de la secuencia de Pátzcuaro, que datan de la década de 1970, sugieren la existencia de bosques de Pinus, Quercus y Alnus. Otro elemento arbóreo presente es el tipo Juniperus, que Watts y Bradbury (1982) asocian a condiciones muy secas, lo que contrasta con la interpretación del registro lacustre con niveles altos (Bradbury, 2000). Los autores resaltan el poco cambio en las comunidades de plantas en Pátzcuaro respecto a otras zonas como la cuenca de México.

En Zacapu continúa la baja tasa de sedimentación y la presencia de ambientes subaéreos, ya que no hay evidencias que permitan suponer la presencia de un cuerpo de agua entre 30000 y 18500 cal AP (25 y 15 ka ${ }^{14} \mathrm{C} \mathrm{AP}$; Metcalfe, 1992; Ortega et al., 2002). Los datos disponibles de la vegetación sugieren la existencia de bosques de Pinus alrededor del lago, y hacia 20000 a 19000 cal AP (17-16 ka ${ }^{14} \mathrm{C} \mathrm{AP}$ ) hay un cambio significativo con el incremento de polen de pastos (Lozano-García y Sosa-Nájera, datos inéditos).

5.3 Glacial Tardío (GTA): 18000-15000 cal AP (14.8-12.7 ${ }^{14} \mathrm{C} \mathrm{ka} \mathrm{AP)}$

En el registro del Jalapasquillo el control cronológico impide ubicar esta ventana de tiempo correctamente, sin embargo se puede identificar que el registro está dominado por polen de Pinus, llegando en algunos niveles al 98\%.

En la cuenca de México, los sitios del norte (Tecocomulco y Texcoco) reflejan eventos de marcada sequía con hiatos sedimentarios. En Tecocomulco se tiene un hiato de sedimentación entre aproximadamente 18500 y 3500 cal AP (15 y $\left.3.3 \mathrm{ka}{ }^{14} \mathrm{C} \mathrm{AP}\right)$. En Texcoco la secuencia litoral de Tepexpan muestra la transición de una sedimentación lacustre a una subaérea con la formación de paleosuelos, lo que refleja la disminución del nivel lacustre antes de los 11500 cal AP (10 ka ${ }^{14} \mathrm{C}$ AP; Sedov et al., 2009b). Los fechamientos disponibles no permiten definir con precisión este momento pero, por correlación con los otros registros del centro del lago, se considera que se encuentra dentro de esta ventana de tiempo, hacia los $17500 \mathrm{cal}$ AP (14.5 ka ${ }^{14} \mathrm{C}$ AP). En la secuencias del centro del lago de Texcoco, la preservación de diatomeas es pobre y existe un hiato sedimentario entre aproximadamente los 17500 y $7000 \mathrm{cal}$ $\mathrm{AP}\left(14.5\right.$ y $\left.6.1 \mathrm{ka}{ }^{14} \mathrm{C} \mathrm{AP}\right)$. En los diagramas polínicos justo antes del hiato el elemento dominante es Pinus.

En Chalco se registra un leve incremento en el nivel lacustre entre 16800 y 15000 cal AP (14 y 12.5 ka ${ }^{14} \mathrm{C}$ AP), con el establecimiento de un conjunto de diatomeas dominado por pequeñas Fragilariales que indican condiciones someras de agua dulce. En el registro de polen entre 18000 y 16800 cal AP (15 y 14 ka ${ }^{14} \mathrm{C}$ AP) aún hay evidencias de comunidades boscosas muy abiertas donde el componente herbáceo tiene valores hasta del 60\%. Posteriormente entre $c a .16800$ y 15000 cal AP (ca. 14 y $12.5 \mathrm{ka}{ }^{14} \mathrm{C}$ AP) se detectan cambios en la composición polínica, con un incremento en el polen arbóreo a partir de la cual se infiere el desarrollo de bosques más cerrados; también hay evidencias de bosques con elementos mesófilos. Aunque Picea está presente, tiene valores más bajos que en el periodo previo.

En la cuenca alta del río Lerma (Chignahuapan) la erosión permanece relativamente baja y el nivel del lago un poco más alto, con la presencia de Cyclotella spp. entre los 19100 y $13000 \mathrm{cal}$ AP (16 y $\left.11 \mathrm{ka}{ }^{14} \mathrm{C} \mathrm{AP}\right)$. Al inicio de esta ventana de tiempo, ca. $18000 \mathrm{cal}$ AP $\left(14.8 \mathrm{ka}{ }^{14} \mathrm{C} \mathrm{AP}\right)$ hay un incremento en polen de Pinus, Quercus, Alnus y tipo Cupressaceae que es seguido por una tendencia posterior hacia el desarrollo de bosques más abiertos; dicha tenencia culmina con un máximo de pastos a los ca. 15000 cal AP (12.5 ka ${ }^{14} \mathrm{C}$ AP). Hay evidencias de condiciones frías y probablemente secas que permitieron un descenso en la línea arbolada. En los conjuntos polínicos hay valores hasta del 
$75 \%$ correspondiente a polen de pastos, baja concentración de polen y un incremento de los elementos de pantano con ciperáceas e Isoëtes. El conjunto de indicadores sugiere ambientes fríos y más secos que los actuales (Lozano-García et al., 2005).

En la parte occidental de la FVTM en el lago de Cuitzeo continúan las tasas de sedimentación muy bajas con la posible presencia de un hiato de sedimentación entre 21000 y 10000 cal AP (17.6 a $8.8 \mathrm{ka}{ }^{14} \mathrm{C}$ AP). En Pátzcuaro, de acuerdo con Bradbury (2000), las condiciones permanecen mas o menos iguales al UMG hasta los $13000 \mathrm{cal}$ AP (11.1 ka ${ }^{14} \mathrm{C}$ AP), con un lago frío y profundo que posiblemente congelaba durante el invierno; sin embargo los valores de Isoëtes son altos en este periodo, lo que parece contradecir la interpretación de un lago profundo. Durante este periodo los valores de los Pinus disminuyen y el tipo Juniperus que es considerado por Watts y Bradbury como indicador de condiciones xéricas aumenta a los $c a .18000 \mathrm{cal}$ AP (Watts y Bradbury, 1982; Bradbury, 2000); posteriormente hay un incremento de Alnus que puede estar relacionado con procesos de regeneración del bosque.

En el lago de Zirahuén el registro paleolimnológico inicia a los $17000 \mathrm{cal} \mathrm{AP}\left(14 \mathrm{ka}{ }^{14} \mathrm{CAP}\right)$, el tipo de diatomeas y la presencia de carbonatos indican que se trataba de un lago somero, levemente alcalino. En el registro de polen se reconstruyen ambientes menos húmedos que el actual con bosques de Pinus alrededor del lago y extensas zonas de pantano donde crecían varias especies de Isoëtes; el conjunto sugiere un ambiente más frío y seco que el presente (Ortega et al., 2010; Torres-Rodríguez, 2009).

En Zacapu, la cronología es pobre y hay evidencias de actividad volcánica, el registro de diatomeas indica niveles lacustres bajos y el decremento de carbono orgánico y la susceptibilidad magnética sugieren condiciones particularmente secas, después de los 16700 y hasta los 5500 cal AP (14 a 4.8 ka ${ }^{14} \mathrm{C}$ AP; Metcalfe, 1992 y 1995; Ortega et al., 2002).

\subsection{Glacial Terminal (GTE): 15000-12000 cal AP (12.7-} $10{ }^{14} \mathrm{C}$ ka AP)

En la zona oriental de la FVTM, poco antes de $c a .10000$ cal AP se presenta el cambio más marcado en la secuencia Tlaloc II, localizada en el cráter del volcán La Malinche, con el paso de un registro dominado por porcentajes muy altos de polen no arbóreo, indicador de que el sito estaba rodeado por zacatonales, a otro en el que se incrementa el polen arbóreo, que refleja una migración hacia altitudes mayores de los bosques de Pinus hatwegii (Straka y Ohngemach, 1989).

En la cuenca de México, los registros se caracterizan por hiatos sedimentarios, en Tecocomulco entre 18500 y $3500 \mathrm{cal}$ AP (15 y $3.3 \mathrm{ka}{ }^{14} \mathrm{C}$ AP), en Texcoco entre 17500 y $7000 \mathrm{cal} \mathrm{AP}\left(14.5\right.$ y $\left.6.1 \mathrm{ka}{ }^{14} \mathrm{C} \mathrm{AP}\right)$ y en Chalco se presenta la depositación de una capa de diatomita con una tasa de sedimentación baja entre los 15000 y 14000 cal AP (12.8 y $\left.10 \mathrm{ka}{ }^{14} \mathrm{C} \mathrm{AP}\right)$ cuya flora de diatomeas sugiere una leve reducción del nivel lacustre con respecto a la etapa anterior. En el polen de Chalco hay un cambio importante en la composición de los conjuntos polínicos, se expanden los bosques de Pinus y Alnus y se reduce la presencia de Cupressaceae; hay, además, entre 13000 y 12000 cal AP (11 y $10 \mathrm{ka}{ }^{14} \mathrm{C} \mathrm{AP}$ ), la presencia de esporas de helechos que pueden estar relacionadas a la inestabilidad del ambiente por el aumento en temperatura y los procesos de sucesión que probablemente ocurrieron en la vegetación durante esta época.

En la cuenca alta del río Lerma (Chignahuapan) los niveles lacustres generalmente se mantienen similares al intervalo anterior hasta la caída de la Pómez Toluca Superior a los $13500 \mathrm{cal}$ AP $\left(11.5 \mathrm{ka}{ }^{14} \mathrm{C} \mathrm{AP}\right)$, producida por el Nevado de Toluca; sólo se detecta un evento puntual seco a los $14300 \mathrm{cal}$ AP $\left(12.4 \mathrm{ka}{ }^{14} \mathrm{C} \mathrm{AP}\right)$ evidenciado por la presencia de hematita y que correlaciona con expansión de pastizales. Después de la caída de la Pómez Toluca Superior se produce un cambio hacia un lago más somero y alcalino. Los datos palinológicos indican que continúan existiendo bosques de coníferas abiertos antes de la emisión de la Pómez Toluca Superior.

En la porción oeste de la FVTM, en el lago de Pátzcuaro Isoëtes comienza a disminuir a partir de los $15000 \mathrm{cal}$ AP y desaparece a los $13000 \mathrm{cal}$ AP; el lago experimenta un cambio marcado por la reducción en las especies planctónicas y el aumento en las Fragilariales después de 13000 cal AP indicando una disminución en el nivel lacustre (Bradbury, 2000). La persistencia de S. oregonicus se interpreta como la persistencia de lluvias en invierno, con una tendencia a ambientes más secos que indican que la lluvia en verano todavía no se activa totalmente, aunque el lago mantuvo niveles más altos que los actuales (Metcalfe et al., 2007). Los conjuntos polínicos indican que los bosques de Pinus se expanden concomitantemente con la disminución de Alnus. Los estudios de vegetación en Michoacán señalan que Alnus accuminata crece rápidamente formando bosques secundarios en espacios que quedan dentro del bosque. Posiblemente el cambio en temperatura del glacial tardío promovió procesos de sucesión en las comunidades boscosas de la cuenca de Pátzcuaro. La presencia de polen de plantas herbáceas como Ambrosia y Artemisia es interpretada como señal de condiciones secas entre 14000 y $13000 \mathrm{cal} \mathrm{AP} \mathrm{(Bradbury,} \mathrm{2000).}$

En Zirahuén se presenta un cambio importante después de los 15000 cal AP con un recambio de especies de diatomeas dominantes (de Cocconeis a Cyclotella-Ulnaria) al mismo tiempo que cesa la precipitación de carbonatos, lo que sugiere un pequeño aumento en el nivel lacustre, mayor productividad y menor erosión, condiciones que persisten hasta $12600 \mathrm{cal}$ AP. En el diagrama polínico entre 15000 y $14000 \mathrm{cal}$ AP (12.7 a $12 \mathrm{ka}{ }^{14} \mathrm{C}$ AP) el único elemento de bosque es Pinus, pero $c a .13500 \mathrm{cal}$ AP $\left(11.5 \mathrm{ka}{ }^{14} \mathrm{CAP}\right)$ hay un cambio en la composición con un incremento en Quercus y Alnus. Hay un aumento en el polen de pastos y de Pinus a los $13000 \mathrm{cal} \mathrm{AP}\left(11.1 \mathrm{ka}{ }^{14} \mathrm{C} \mathrm{AP}\right)$ y posteriormente inicia 
un cambio en la composición de las comunidades con un aumento de Quercus y Alnus; éste último se reporta como un árbol de rápido crecimiento posterior a la perturbación del bosque, indicando el proceso de sucesión, una señal muy parecida a la observada en el lago de Pátzcuaro (Ortega et al., 2010). En Zacapu el registro indica condiciones secas hasta los $5500 \mathrm{cal}$ AP (4.8 ka ${ }^{14} \mathrm{CAP}$; Metcalfe, 1992 y 1995; Ortega et al., 2002).

\section{Discusión}

Con base en la recopilación de datos paleolimnológicos y de paleo-vegetación expuestos, los sitios con un registro temporal más largo como son Tecocomulco, Texcoco, Chalco y Cuitzeo presentan una tendencia hacia menores niveles lacustres que se establece en tiempos anteriores al GTM, alrededor de los 30000 AP, y continúa durante el GTM. Para este periodo no hay evidencias de avances glaciales.

Para el UMG se documentan los avances glaciales más importantes de los últimos 50000 años. Así, el avance Hueyatlaco-1 implica una disminución de temperatura del orden de 6 a $7.5^{\circ} \mathrm{C}$ (Lachniet y Vázquez-Selem, 2005; Tabla 2).

Dados los contrastes topográficos que caracterizan a la FVTM, las variaciones en temperatura y humedad de una zona a otra debieron afectar la distribución y composición de la vegetación que se distribuye en el área. Los datos palinológicos son una fuente de información sobre los cambios en la vegetación, así como el desplazamiento en la posición altitudinal de la línea arbolada. Para el Iztaccíhuatl, datos previos indican que durante el Holoceno temprano la línea arbolada se estableció 500 a $700 \mathrm{~m}$ por debajo de su posición moderna (4020 msnm) (Lozano-García y Vázquez-Selem, 2005). Con esta información es posible estimar el desplazamiento de la vegetación montana durante los periodos previos como el UMG y el GTA. De acuerdo con Körner y Paulsen (2004) el límite superior del bosque corresponde a la isoterma media del suelo de $6.7^{\circ} \mathrm{C}$, que parece definir el establecimiento de la línea arbolada de altitud a nivel global. Utilizando la reconstrucción de la temperatura con base en los cambios en la altitud de la ALE, es posible estimar la posición del límite superior de la vegetación alpina y el límite superior del bosque (Figuras $2,3,4)$.
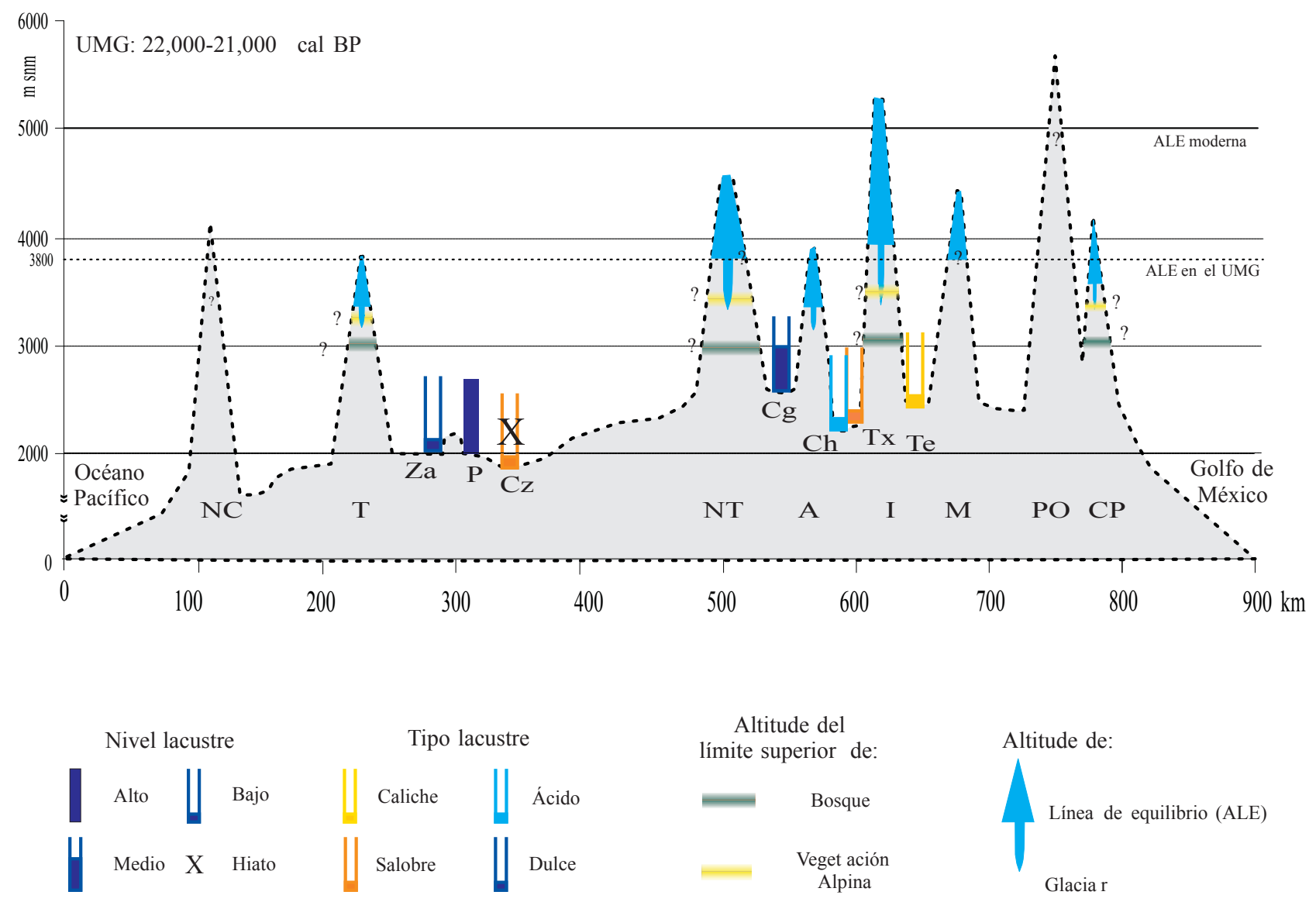

Figura 2. Niveles y tipos lacustres; posición altitudinal del límite superior del bosque y vegetación alpina; altitud de la línea de equilibrio (ALE) y avances glaciares en diversos sitios a lo largo de la FVTM durante el UMG (22,000 - 21,000 cal AP). Montañas y lagos como en la Figura 1. 

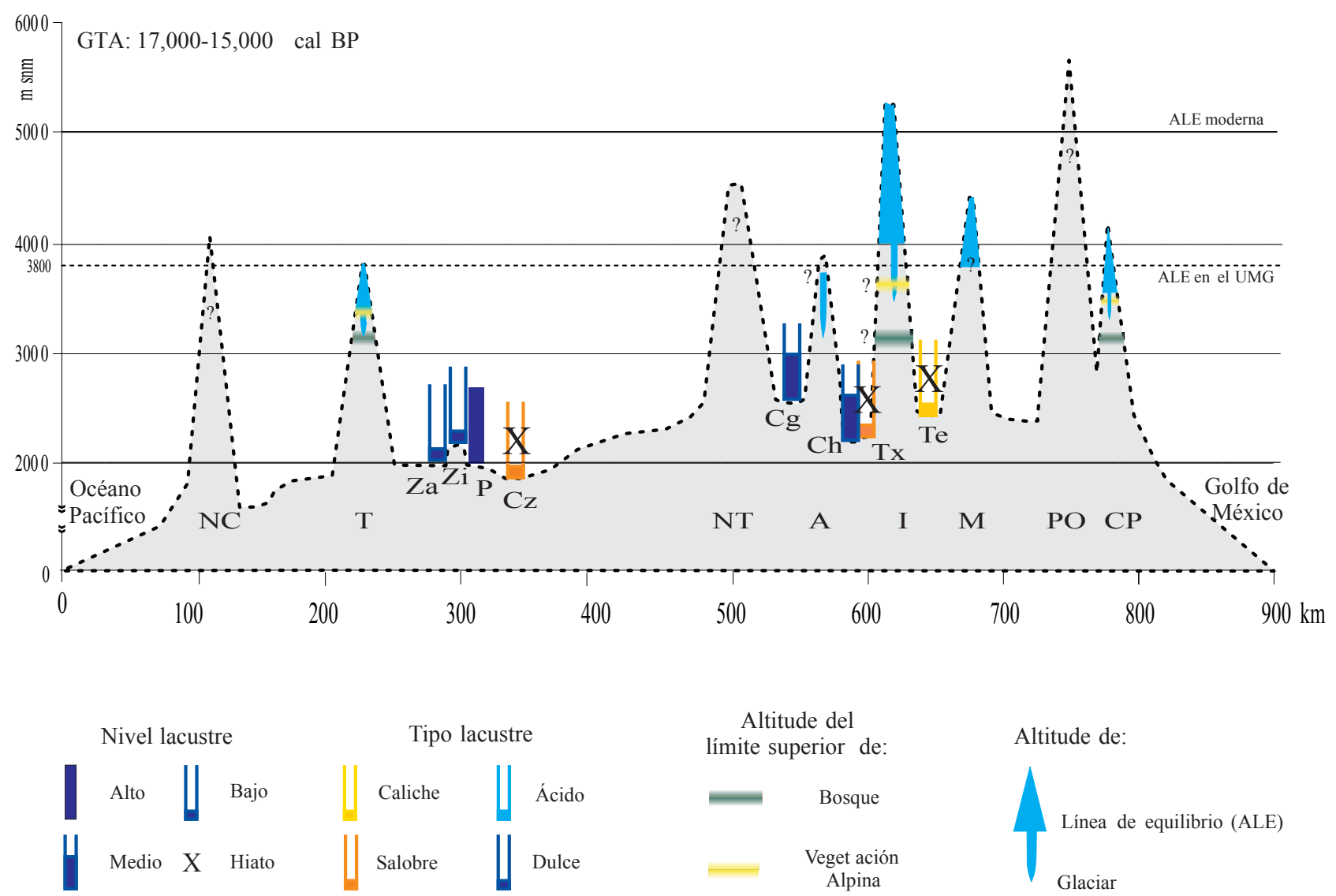

Figura 3. Niveles y tipos lacustres, posición altitudinal del límite superior del bosque y vegetación alpina y altitud de la línea de equilibrio (ALE) y avances glaciares en diversos sitios a lo largo de la FVTM durante el GTA (17,000 - 15,000 cal AP). Montañas y lagos como en la Figura 1.

La FVTM ha sido considerada como un área que, por su evolución geológica, ofrece gran variedad de hábitats para las numerosas especies de Pinus. Con los datos que aquí se resumen sobre el cambio climático de los últimos miles de años es probable que no sólo los microhábitats que surgieron por la actividad volcánica han contribuido a los procesos de especiación, sino también que el descenso de las franjas de vegetación de hasta $c a .1000$ m contribuyó a la diversificación de los Pinus en México. En la FVTM la riqueza de especies de Pinus es muy alta: de las $\sim 100$ especies de Pinus que existen, la mitad se encuentran en México, establecidas en rangos amplios de temperatura (15 $\left.{ }^{\circ} \mathrm{C} \pm 10^{\circ} \mathrm{C}\right)$ y precipitación $(800 \pm 150 \mathrm{~mm}$; Velázquez et al., 2000), la mayor parte de ellas en las zonas montañosas. De manera similar existe una gran diversidad de especies de Quercus y los cambios en temperatura y precipitación del Pleistoceno tardío causaron desplazamientos altitudinales y latitudinales, fragmentando y/o ampliando los rangos de distribución.

En la Figura 5 se muestran los rangos actuales de la vegetación montana del centro de México y el modelo del desplazamiento probable de la vegetación durante el UMG de $c a .1000 \mathrm{~m}$. La migración hacia menores altitudes y la probable expansión de la vegetación montana se reflejan, en la mayor parte de los diagramas polínicos de las cuencas, en una clara dominancia del polen de Pinus. En Oriental se presentan valores muy altos ( 80 a $90 \%$ ) de Pinus, mientras que en las cuencas de menor altitud como Chalco y Texcoco este desplazamiento de las franjas repercute en porcentajes menores de polen de Pinus ( 40 a $60 \%$ ), aunque siempre son el elemento dominante de los conjuntos arbóreos en los diagramas de polen. Otra característica de los conjuntos polínicos durante el glacial son los valores de polen de pastos más elevados que durante el Holoceno. En la cuenca del río Lerma ( $2600 \mathrm{msnm}$ ), la de mayor altitud, esta tendencia es más marcada y la dominancia de los pastos llega al 60\%, lo cual se interpreta como la existencia de bosques muy abiertos y el descenso de los zacatonales en respuesta al enfriamiento del UMG (Lozano-García et al., 2005). Estas evidencias palinológicas sugieren que para el periodo de máximo frío en el centro de México, ocurrió una expansión de los bosques predominantemente de Pinus, más abiertos y con un componente herbáceo importante. Esta misma diversidad de hábitats posiblemente permitió que ciertas zonas de mayor humedad, como cañadas, albergaran elementos mesófilos como Podocarpus y Abies entre otros. En los registros palinológicos se detecta, en muy bajas proporciones, polen de Picea; esta conífera es un taxa 


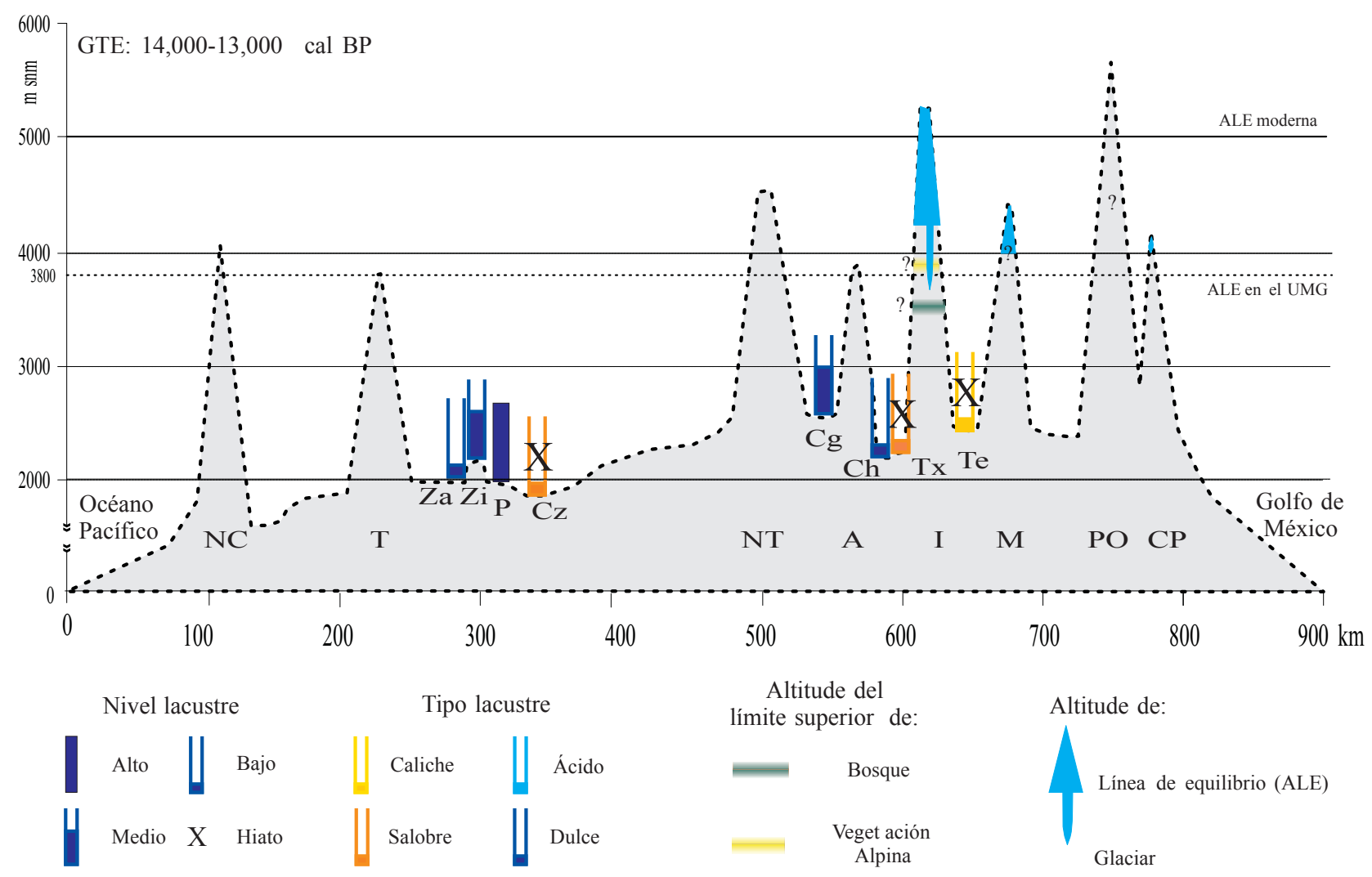

Figura 4. Niveles y tipos lacustres, posición altitudinal del límite superior del bosque y vegetación alpina y altitud de la línea de equilibrio (ALE) y avances glaciares en diversos sitios a lo largo de la FVTM durante el GTE (14000 - 13000 cal AP). Montañas y lagos como en la Figura 1.

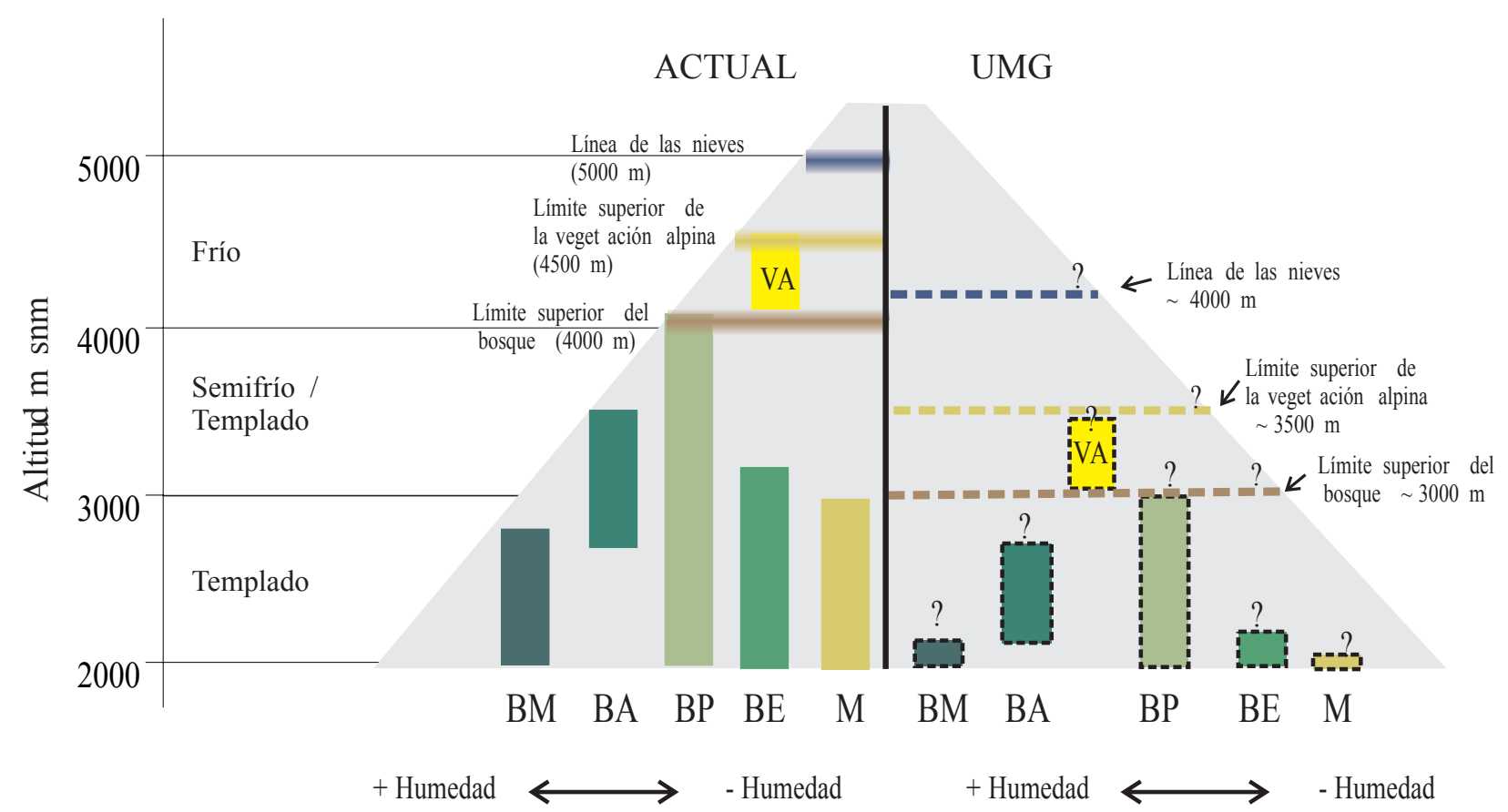

Figura. 5 Esquema de rangos altitudinales actuales y durante el UMG de la vegetación montana en el centro de México, ordenada a lo largo del gradiente de temperatura. Las líneas punteadas corresponden a la posición probable de la vegetación montana para el UMG con base en un descenso del límite de las nieves de $\sim 1000 \mathrm{~m}$ y en una disminución de la temperatura de $6{ }^{\circ} \mathrm{C}$. Se muestra el límite superior de la vegetación alpina y del bosque. BA: bosque de Abies (oyamel); BM: bosque mesófilo; BP: bosque de Pinus (pino); BE: bosque de Quercus (encino); VA: vegetación alpina; M: matorral. 
boreal que en la actualidad se encuentra en bosques fríostemplados del norte del país en pequeñas poblaciones en altitudes que van de los 2000 a los $3700 \mathrm{msnm}$. Su presencia en las secuencias polínicas del centro indica la expansión hacia el sur, y en consecuencia la existencia de climas fríos.

Durante el UMG, la ALE de los glaciares se encuentra a menor altitud en montañas cercanas a las costas (Tancítaro, Cofre de Perote) que en el Iztaccíhuatl, que es una montaña 1000 m más alta situada en el interior de la FVTM (Figura 2). Asumiendo que el principal control de los glaciares es la temperatura (Ohmura et al., 1992) y que durante el UMG las temperaturas se encontraban abatidas de manera similar en toda la región, cabe pensar que tales variaciones en la ALE se deben a un gradiente de precipitación de las costas hacia el interior. Por ello, los glaciares en montañas del interior (Iztaccíhuatl) no descendieron tanto como en montañas con mayor influencia marítima. En la misma lógica, la menor altitud de la ALE en el Tancítaro respecto al Cofre de Perote indica mayor aporte de humedad desde el Pacífico que desde el Golfo de México durante la misma fase. Lo anterior sugiere que durante el UMG hubo cierta variabilidad climática regional en el centro de México que determinó ambientes relativamente más húmedos en los extremos oriental y occidental de la FVTM versus el centro, donde todo indica que se desarrollaron ambientes menos húmedos.

Si bien no hay datos sobre la paleovegetación para toda la FVTM que permitan detectar este gradiente de humedad, es posible observar en el registro polínico indicios de condiciones secas para algunas cuencas durante el UMG. Tal es el caso de Chalco en el sector sur de la cuenca de México, dónde se registra la presencia abundante de polen de Mimosa aff. biuncifera, el cual sugiere ambientes secos, justo en el entorno del Iztaccíhuatl. Otro elemento que es más abundante durante el UMG en los registros polínicos de Pátzcuaro, Chalco, Texcoco y Lerma, es el tipo Cupressaceae (Cupressus, Juniperus), el cual está asociado a ambientes secos. Los cambios de las temperaturas estacionales, la magnitud y la estacionalidad de la precipitación así como la insolación modificaron la cubierta vegetal en esta amplia zona de México. La presencia de ciertos taxa como Mimosa y Poacaeae con abundancias importantes en los conjuntos polínicos del máximo glacial o la presencia de Picea sugieren la existencia de comunidades diferentes (no-análogas) a las actuales.

A pesar de la discontinuidad de los registros de niveles lacustres durante el UMG, en general los datos paleolimnológicos indican niveles disminuidos o francamente bajos, en concordancia con la interpretación de climas más secos derivada de los datos palinológicos. La presencia de ambientes más secos que los actuales en el centro y este de la FVTM puede explicarse por la migración al sur de la ZITC y de los vientos alisios, principal fuente de humedad para la región. Sin embargo, los datos del lago de Pátzcuaro y, hasta cierto punto, los de Cuitzeo, en la zona centro-oriente de la FVTM contrastan al indicar niveles lacustres altos durante este intervalo (o en el caso de Cuitzeo, por lo menos durante una parte de este intervalo, Figura 2b). Bradbury $(1997,2000)$ explica la disparidad del patrón de niveles lacustres de Pátzcuaro durante el UMG en términos de diferencias en las fuentes de aporte de humedad. El efecto de la migración al sur de los vientos del oeste durante el UMG es posible detectarlo en los registros paleoecológicos del norte de México, donde hay evidencias de cambios significativos en los ecosistemas. En la región de los actuales desiertos Sonorense y Chihuahuense se desarrollaron de bosques de Juniperus y pinos piñoneros y existieron amplias extensiones lacustres y zonas inundables asociadas al aumento de la precipitación de invierno (Metcalfe, 2006). Bradbury (1997) sugiere que durante el UMG el incremento de humedad en Pátzcuaro se derivó del desplazamiento al sur de los vientos del oeste a latitudes tan meridionales como el centro-oeste de la FVTM. Este mecanismo es afín al descenso de la ALE registrado en el Tancítaro; sin embargo, no explica las condiciones secas que prevalecieron en la cuenca de Zacapu o la muy baja tasa de sedimentación en Cuitzeo entre 21000 y 10000 cal AP. Según estas tendencias, se puede marcar un contraste entre la parte este y oeste de la FVTM. Al respecto es interesante notar que Frederick (1995) estimó una disminución de la precipitación de $\sim 40 \%$ durante el UMG y el GTA para el sur del estado de Guanajuato, con base en estratigrafía y sedimentología aluvial, suelos e isótopos estables de carbono.

La interpretación que realiza Bradbury (2000) en la que considera que Pátzcuaro pudo haberse congelado estacionalmente durante el UMG y el GTA parece poco factible dado que esto requeriría un descenso en la temperatura de mas de $12{ }^{\circ} \mathrm{C}$, fuera del rango estimado por el descenso de la ALE. Esta condición sólo podría ser probable para la cuenca de mayor elevación, la cuenca alta del Lerma (Chignahuapan), donde un enfriamiento de $9^{\circ}$ a $10^{\circ} \mathrm{C}$ sería compatible con temperaturas levemente por debajo de $0{ }^{\circ} \mathrm{C}$ durante los meses más fríos del año. Sin embargo, hoy en día los lagos dentro del cráter del Nevado de Toluca, a una altitud de $4200 \mathrm{msnm}$ (y con una temperatura media $\sim 10^{\circ} \mathrm{C}$ menor que la de Chignahuapan), se congelan excepcionalmente en invierno.

Otra fuente de precipitación que en la actualidad aporta humedad durante el invierno en el centro-oriente de México son los nortes. Se ha sugerido un incremento en la frecuencia e intensidad de nortes durante el UMG, lo que pudo aumentar la precipitación en invierno en esta región (Lozano-García et al., 2007). Este mecanismo explica el desarrollo de los glaciares en las montañas, en un ambiente frío con menor precipitación de verano debido a que la migración de la ZITC al norte se debilitó y, con la reducción de la estacionalidad y la temperatura, se favoreció una menor evapotranspiración. Este mecanismo ha sido utilizado para explicar los efectos de la "Pequeña Edad de Hielo" en la zona tropical de Los Tuxtlas (Lozano-García et al., 2007) y en el registro de Peten Itzá donde se documentan 
condiciones húmedas durante el UMG asociadas a una menor estacionalidad e inviernos más húmedos (Hodell et al., 2008).

Durante el GTA (18000-15000 cal AP) los glaciares en el Iztaccíhuatl se contrajeron ligeramente (ascenso de la ALE de $\sim 100 \mathrm{~m}$ ), pero aparentemente en montañas cercanas a la costa se mantuvieron en posiciones similares a las del UMG. Desde la sola perspectiva de los glaciares esto puede explicarse por una intensificación del gradiente de humedad de las costas hacia el interior, es decir por condiciones relativamente menos húmedas al interior. Cabe mencionar que esto no es contradictorio con la presencia de glaciares, ya que éstos son mucho más sensibles a la temperatura que a la precipitación. Las relaciones climaALE de Ohmura et al. (1992) muestran que a temperaturas como las que caracterizan la ALE en montañas tropicales (cercanas a $0{ }^{\circ} \mathrm{C}$ ), es posible la existencia de glaciares con una precipitación incluso 50\% menor que la actual.

En concordancia con lo anterior, la señal polínica durante el GTA en general es similar a la del UMG. Durante el GTA prevalecieron los niveles lacustres bajos, lo que sugiere que continúa la depresión en los sistemas climáticos (alisios y ZITC) que aportan lluvias de verano a esta región, si bien a lo largo de esos 3000 años las señales no apuntan en la misma dirección en toda la región. Así por ejemplo, en Chignahuapan y Chalco se registra una leve recuperación del tirante de agua. Las tendencias en Texcoco y Tecocomulco no son conocidas, pues existe un hiato de sedimentación, pero es probable que el hiato guarde relación con las condiciones climáticas de inicios del Holoceno. En Chalco y en la cuenca alta del río Lerma se registra un leve aumento en el nivel lacustre, lo que apoya la idea de que los hiatos registrados en Texcoco y Tecocomulco estén relacionados con las condiciones climáticas del Holoceno temprano.

Durante el GTE (15000 a 12000 cal AP) ocurren cambios significativos dado que los glaciares en todas las montañas se encuentran en retroceso, reflejando un incremento en la temperatura cercano a $2{ }^{\circ} \mathrm{C}$ durante ese lapso. En la señal polínica de todos los sitios estudiados se identifica una expansión de los bosques, probablemente relacionada con la migración hacia mayores altitudes en respuesta al incremento en la temperatura. En los niveles lacustres se manifiestan nuevamente las discrepancias entre los registros de las cuencas del centro-oeste de la FVTM, ya que se registra un incremento de nivel en Zirahuén pero en el lago de Pátzcuaro se produce una reducción en el nivel lacustre. Bradbury (2000) lo interpreta como una disminución de las lluvias de invierno debido a la migración hacia el norte de los vientos del oeste y el gradual establecimiento de los climas modernos con lluvia estival asociada a los vientos alisios. Zacapu y Cuitzeo, mantienen niveles bajos con hiatos sedimentarios.

Los registros paleopedológicos del centro de México coinciden en que antes y durante el GTM prevalecieron condiciones óptimas para la pedogénesis en las montañas y cuencas del centro de México, incluso a más de 3000 msnm (Heine, 1975, 1978; Sedov et al., 2001, 2003b, 2009a; Solleiro-Rebolledo et al., 2006). Para el UMG, en cambio, las evidencias son contradictorias. En Tlaxcala, a $2600 \mathrm{msnm}$, Sedov et al. (2009a) encuentran una pedogénesis compatible con condiciones frías y de humedad continua a lo largo del año, si bien la mayor parte de los fechamientos presentados indican edad anteriores al UMG. En Teotihuacan, a $2950 \mathrm{msnm}$, los paelosuelos indican ambientes húmedos $\mathrm{y}$ fríos antes del UMG 25000 cal $\mathrm{BP}$ $\left(\sim 22.0{ }^{14} \mathrm{C} \mathrm{ka}\right)$ que parecen mantenerse durante UMG y el GTA 22000 cal BP a 15800 cal BP $\left(18.7\right.$ a $\left.13.3{ }^{14} \mathrm{C} \mathrm{ka}\right)$, aunque con condiciones cada vez más secas al final de su desarrollo (Solleiro-Rebolledo et al., 2006). Una tendencia hacia condiciones progresivamente más secas durante y después del UMG podría explicar el escaso registro de suelos en el Nevado de Toluca ( $\sim 3000 \mathrm{msnm})$ entre 30500 cal BP y 13400 cal BP $\left(\sim 24{ }^{14} \mathrm{C}\right.$ ka y $11.6{ }^{14} \mathrm{C} \mathrm{ka}$; Sedov et al., 2003a,b). En concordancia con lo anterior, para Heine (1984, 1989) los paleosuelos de La Malinche indican condiciones húmedas antes del UMG, pero durante todo el UMG y hasta $\sim 14000$ cal AP $\left(\sim 12{ }^{14} \mathrm{C} \mathrm{ka}\right)$ se establecieron condiciones de aridez que detuvieron el desarrollo de suelos en todos los pisos altitudinales.

\section{Consideraciones finales}

A partir de la revisión de los registros glaciales, se puede concluir que el máximo avance de los glaciares de fines del Pleistoceno en las montañas del centro de México ocurrió entre 21000 y 17500 cal AP, esto es, dentro del UMG planetario situado entre 26500 y 20000-19000 cal AP (Clark et al., 2009). El descenso estimado de la ALE de 1000 $\mathrm{m}$ permite estimar un enfriamiento de entre $6^{\circ}$ y $8^{\circ} \mathrm{C}$. La respuesta de las comunidades de plantas al enfriamiento se identifica en los registros polínicos como un desplazamiento en los rangos de distribución hacia altitudes menores. El clima del UMG parece haber causado modificaciones en la composición de las comunidades favoreciendo a las especies más resistentes a las nuevas condiciones ambientales. Sin embago, la vegetación no sólo responde al enfriamiento, sino también al cambio en la humedad, dado que hay evidencias de climas relativamente secos a pesar de la disminución en temperatura y, por lo tanto, de evaporación. La expansión de los glaciares durante el UMG no es incompatible con una disminución de la precipitación, ya que los glaciares dependen primordialmente de temperaturas bajas (ascenso de la ALE) y, en menor medida, de la precipitación. Glaciares como los del Iztaccíhuatl durante el UMG pueden haberse formado con una precipitación sustancialmente menor a la actual, lo cual es compatible con evidencias paleoecológicas de menor humedad.

Los cambios observados no son iguales a todo lo largo del transecto E-W de la FVTM, pues hay variaciones dependiendo de la localización y la altura de la cuenca, lo cual es indicativo de una cierta variabilidad climática 
regional, aunque con los registros disponibles no es fácilmente detectable. La integración de evidencias paleolimnológicas y de avances glaciares, a pesar de las dificultades antes mencionadas, sugiere que hubo variaciones en los aportes de humedad entre los sitios estudiados. Varios registros paleolimnológicos y polínicos apoyan condiciones más secas (y frías) en el centro y centro-oriente de México durante el UMG, en particular entre 21000 y 18000 cal AP. Sólo el registro de Pátzcuaro indica condiciones relativamente más húmedas, lo que se ha interpretado como un mayor aporte de humedad por los vientos del oeste (Bradbury, 1997) y la alteración del monzón mexicano, afectado por el desplazamiento latitudinal de la ZITC a una posición más al sur.

En latitudes templadas la deglaciación inició en 2000019000 cal AP (Clark et al., 2009). El hecho de que en las montañas de la FVTM las ALEs hayan permanecido my próximas a las del UMG hasta $\sim 15000$ cal AP sugiere que algún mecanismo climático regional mantuvo las temperaturas en valores cercanos a los del UMG hasta ese momento. Lo mismo se aplica a las condiciones paleoecológicas en torno a las cuencas lacustres, que no se modificaron significativamente durante el GTA.

La deglaciación pronunciada en las montañas del centro de México inicia entre 15000 y $14000 \mathrm{cal} \mathrm{AP} \mathrm{y} \mathrm{se}$ mantiene hasta hace $\sim 12500 \mathrm{cal} \mathrm{AP}$, indicando un aumento de la temperatura del orden de $\sim 2{ }^{\circ} \mathrm{C}$ en ese lapso. Las comunidades vegetales en torno a las cuencas lacustres reflejan ese incremento térmico, mientras que los niveles lacustres dominantemente bajos (con probables hiatos) parecen indicar que el aumento térmico no fue compensado por un incremento significativo de la precipitación durante esta fase.

Si bien es posible estimar la magnitud del enfriamiento en esta región durante el UMG, la evaluación de las variaciones de humedad presenta dificultades relacionadas al tipo de registro y a la resolución de los datos. Las evidencias analizadas sugieren condiciones menos húmedas que las actuales para el UMG. Sin embargo, con indicadores de mayor sensibilidad y resolución será posible identificar los eventos Heinrich y las oscilaciones D/O, que seguramente alteraron los patrones de precipitación y los niveles lacustres y que tuvieron consecuencias en la biota.

La falta de registros provenientes de cuencas localizadas en los extremos oriental y occidental de la FVTM, impiden hasta ahora explicar las diferencias que se presentan en algunos registros sobre los aportes de humedad durante el UMG. Las problemáticas referentes a la preservación de los indicadores, los eventos volcánicos que alteran los registros, la correcta identificación del material paleontológico, entre otras dificultades, se resolverá con un mayor esfuerzo de investigación en esta zona.

Por su localización geográfica la FVTM es una frontera, por lo que entender y documentar la variabilidad climática de esta región tan importante desde un punto de vista geológico-tectónico, biogeográfico y paleoclimático es fundamental para comprender el futuro cambio ambiental dado que, además, es dónde se encuentra asentada una parte importante de la población de México.

\section{Agradecimientos}

Este trabajo se realizó con el apoyo de los proyectos DGAPA-PAPIIT IN212606, IN220609, IN118109, IN113408, IN107902, IN114906 y CONACYT 50780-F..

\section{Referencias}

Arce, J.L, Cervantes, K.E., Macias, J.L., Mora, J.C., 2005, The 12.1 ka Middle Toluca Pumice: a dacitic plinian-subplinian eruption of Nevado de Toluca in Central Mexico: Journal of Volcanology and Geothermal Research, 147, 125-143.

Bradbury, J.P., 1971, Paleolimnology of Lake Texcoco, México. Evidence from diatoms: Limnology and Oceanography, 16, 180-200.

Bradbury, J.P., 1989, Late Quaternary lacustrine paleoenvironments in the Cuenca de Mexico: Quaternary Science Reviews, 8, 75-100.

Bradbury, J.P., 1997, Sources of glacial moisture in Mesoamerica: Quaternary International, 43/44, 97-110.

Bradbury, J.P., 2000, Limnologic history of Lago de Pátzcuaro, Michoacán, Mexico for the past 48000 years: impacts of climate and man: Palaeogeography, Palaeoclimatology, Palaeoecology, 163, 65-95.

Bush, M.B., Correa-Metrio, A.Y., Hodell, D.A., Brenner, M., Anselmetti, F.S., Ariztegui, D., Mueller, A.D., Curtis, J.H., Grzesik, D.A., Burton, C., Gilli, A., 2009, Re-evaluation of climate change in lowland Central America during the Last Glacial Maximum using new sediment cores from lake Petén Itzá, Guatemala, en Vimeux, F., Sylvestre, F.,Khodri, M., (eds.), Past climate cariability in South America and surrounding regions: Developments in Paleoenvironmental Research, 14, 113-128.

Caballero, M., Ortega, B., 1998, Lake levels since about 40000 years ago at Lake Chalco, near Mexico City: Quaternary Research, 50, 69-79.

Caballero, M., Lozano, G.S., Ortega, B., Urrutia, J., Macías, J.L., 1999, Environmental characteristics of lake Tecocomulco, northern basin of Mexico, for the last 50,000 years: Journal of Paleolimnology, 22, 399-411.

Caballero, M., Macías, J.L., Lozano, G.S., Urrtuia F.J., Bernal, R., 2001, Late Pleistocene-Holocene volcanic stratigraphy and paleoenvironments of the upper Lerma basin, Mexico, in White, J.D.L., Riggs, N.R. (eds.), Volcaniclastic sedimentation in lacustrine settings: International Associaton of Sedimentologists Special Publication, 30, 247-261.

Caballero M., Ortega, B., Valadez, F., Metcalfe, S.E., Macías J.L., Sugiura Y., 2002, Sta. Cruz Atizapán: a 22-ka lake level record and climatic implications for the late Holocene human ocupation in the Upper Lerma basin, Central Mexico: Palaeogeography, Palaeoclimatology, Palaeoecology, 186, 217-235.

Castro-Govea, R., Siebe, C., 2007, Late Pleistocene-Holocene stratigraphy and radiocarbon dating of La Malinche volcano, Central Mexico: Journal of Volcanology and Geothermal Research, 162, 20-42.

Clark, P.U., Dyke, A.S., Shakun, J.D., Carlson, A.E., Clark, J., Wohlfarth, B., Mitrovica, J.X., Hostetler, S.W., McCabe, A.M., 2009, The Last Glacial Maximum: Science, 325, 710-714.

CLIMAP Project members, 1981, Seasonal reconstructions of the Earth's surface at the last Glacial Maximum: Boulder, Colorado, Geological Society of America, $18 \mathrm{p}$.

D'Antonio, M., Capra, L., Sarocchi, D., Bellotti, F., 2008, Reconstruction of the eruptive event associated to the emplacement of the $13 \mathrm{ka}$ El Refugio pyroclastic flow, Nevado de Toluca volcano (Mexico): Revista Mexicana de Ciencias Geologicas, 25, 115-134. 
Farrera, I., Harrison, S.P., Prentice, I.C., Ramstein, G., Guiot, J., Bartlein, P.J., Bonnefille, R., Bush, M., Cramer, W., von Grafenstein, U., Holmgren, K., Hooghiemstra, H., Hope, G., Jolly, D., Lauritzen, S.E., Ono, Y., Pinot, S., Stute, M., Yu, G., 1999, Tropical climates at the Last Glacial Maximum: a new synthesis of terrestrial palaeoclimate data. I. Vegetation, lake-levels and geochemistry: Climate Dynamics, $15,823-856$.

Ferrusquía-Villafranca, I., Arroyo-Cabrales, J., Martínez-Hernández, E., Gama-Castro, J., Ruiz-González, J., Polaco, O.J., Johnson, E., 2010, Pleistocene mammals of Mexico: A critical review of regional chronofaunas, climate change response and biogeographic provinciality: Quaternary International, 217, 53:104.

Frederick, C.D., 1995, Fluvial response to Late Quaternary climate change and land use in central Mexico: Austin, Texas, EUA, The University of Texas at Austin, Tesis doctoral, 304 p.

Gillespie, A., Molnar, P., 1995, Asynchronous maximum advances of mountain and continental glaciers: Review of Geophysics, 33, 311-364.

González-Quintero, L. Fuentes-Mata, M., 1980, El Holoceno de la porción central de la Cuenca de valle de México: Coloquio sobre Paleobotánica y Palinología: Colección Científica, Serie Prehistoria, 86, I.N.A.H., Mexico, Memoria, 3, 113-132.

Heine, K., 1975, Studien zur jünquartären Glazialmorphologie mexikanischer Vulkane. Mit einem Ausblick auf die Klimaentwicklung: Wiesbaden, Hesse, Alemania, Franz Steiner, 178 p.

Heine, K., 1976, Schneegrenzdepressionen, Klimaentwicklung, Bodenerosion und Mensch im zentralmexikanischen Hochland im jüngeren Pleistozän und Holozän: Zeitschrift für Geomorphologie Neue Folge, Supplement Band, 24, 160-176.

Heine, K., 1978, Neue Beobachtungen zur Chronostratigraphie der mittelwisconsin-zeitlichen Vergletscherungen und Böden mexikanischer Vulkane: Eiszeitalter und Gegenwart, 28, 139-147.

Heine, K., 1984, The classical Late Weichselian climatic fluctuations in Mexico, en Morner, N., Karlen, W. (eds.), Climatic changes on a yearly to millenial basis; geological, historical and instrumental records: Dordrecht, Países Bajos, Reidel, 95-115.

Heine, K., 1988, Late Quaternary glacial chronology of the Mexican volcanoes: Die Geowissenschaften, 6, 197-205.

Heine, K., 1989, Die letzteiszeitliche Vergletscherung Mexikanischer Vulkane als Zeugnis hochglazialer Aridität in Mittelamerika: Acta Albertina Ratisbonensia, 46, 93-106.

Heine, K., 1994, Present and past geocryogenic processes in Mexico: Permafrost and Periglacial Processes, 5, 1-12.

Hernández-Cerda, M.E., Carrasco-Anaya, G., 2007, Rasgos climáticos más importantes, in Luna, I., Morrone, J.J., Espinosa, D. (eds.), Biodiversidad de la Faja Volcánica Transmexicana, Universidad Nacional Autónoma de México: 57-72.

Hodell, D.A., Anselmetti, F.S., Ariztegui, D., Brenner, M., Curtis, J.H., Gilli, A., Grzesik, D.A., Guilderson, T.J., Müller, A.D., Bush, M.B., Correa-Metrio, Y.A., Escobar, J., Kutterolf, S., 2008, An 85-ka Record of Climate Change in Lowland Central America: Quaternary Science Reviews, 27, 1152- 1165.

Israde, I.,. Garduño, V.H., Ortega, R., 2002, Paleoambiente lacustre del Cuaternario tardío en el centro del lago Cuitzeo: Hidrobiológica, 12, 61-78.

Körner, C., Paulsen, J., 2004, A world-wide study of high altitude treeline temperatures: Journal of Biogeography, 31, 713-732.

Kutzbach, J.E., Guetter, P.J. Behling P.J.,Selin, R., 1993, Simulated climatic changes: results of the COHMAP climate-model experiments, en Wright, H.E., Kutzbeach, J.E. Webb, T., Ruddiman, W.F.,StreetPerrott, F.A., Bartlein, P.J. (eds.), Global climates since the last glacial maximum: Minneapolis, Minnesota, EUA, University of Minnesota Press, 24-93.

Lachniet, M.S., Vázquez-Selem, L., 2005, Last Glacial Maximum equilibrium line altitudes in the circum-Caribbean (Mexico, Guatemala, Costa Rica, Colombia, and Venezuela): Quaternary International, 138-139, 129-144.

Lamb, A.L., Gonzalez, S., Huddart, D., Metcalfe, S.E., Vane, C.H., Pike, A.W.G., 2009, Tepexpan Palaeoindian site, Basin of Mexico: multi- proxy evidence for environmental change during the late Pleistocenelate Holocene: Quaternary Science Reviews, 28, 2000-2016.

Lea, D.W., Pak, D.K., Spero, H.J., 2000, Climate impact of Late Quaternary equatorial Pacific sea surface temperature variations: Science, 289, 1719-1724.

Lorenzo, J.L., 1964, Los glaciares de México: México, D.F., México, Instituto de Geofísica- UNAM, 124 p.

Lozano-García, M.S., 1996, La vegetación Cuaternaria en el Centro de México: Registros Palinológicos e Implicaciones Paleoclimáticas: Boletín de la Sociedad Botánica de México, 58, 113-127.

Lozano-García, M.S., Ortega-Guerrero, B., 1994, Palynological and magnetic susceptibility records of Lake Chalco, central Mexico: Palaeogeography, Palaeoclimatology, Palaeoecology, 109, 177-191.

Lozano-García, M.S., Ortega-Guerrero, B., 1998, Late Quaternary environmental changes of the central part of the Basin of Mexico; correlation between Texcoco and Chalco sub-basins: Review of Palaeobotany and Palynology, 99, 77-93.

Lozano-García, M.S., Vázquez-Selem, L., 2005, A High Elevation Holocene Pollen Record from Iztaccihuatl volcano, Central México: The Holocene, 15 (3), 329-338.

Lozano-García, M.S., Ortega-Guerrero, B., Caballero, M., UrrutiaFucugauchi, J., 1993, Late Pleistocene and Holocene paleoenvironments of the Chalco Lake, Central Mexico: Quaternary Research, 40, 332-342.

Lozano-García, S., Sosa-Najera, S., Sugiura, Y., Caballero, M., 2005, 23000 $\mathrm{yr}$ of vegetation history of the Upper Lerma, a tropical high-altitude basin in Central Mexico: Quaternary Research, 64, 70-82.

Lozano-García, M.S., Caballero, M., Ortega, B., Rodríguez, A., Sosa, S., 2007, Tracing the effects of the Little Ice Age in the tropical lowlands of eastern Mesoamerica: Proceedings of the National Academy of Sciences, 104, 16200-16203.

Machain-Castillo, M.L., Arellano-Torres, E., Cuesta-Castillo, B., en prensa, Modificación del patrón de vientos y surgencias en el golfo de Tehuantepec durante el último máximo glacial: Escenarios de cambio ambiental, registros del Cuaternario en América Latina: México, D.F., Fomento Editorial Universidad Nacional Autónoma de México.

Metcalfe, S.E., 1992, Changing environments of the Zacapu basin, Central Mexico: a diatom-based history spanning the last 30000 years: Oxford, Inglaterra, University of Oxford Research Paper No. 48,38 p..

Metcalfe, S.E., 1995, Holocene environmental change in the Zacapu Basin, Mexico: a diatom-based record: The Holocene, 5, 196-208.

Metcalfe, S.E., 2006, Late Quaternary environments of the northern deserts and Central Transvolcanic Belt of Mexico: Annals of the Missouri Botanical Garden, 93, 258-273.

Metcalfe, S.E., O'Hara, S.L., Caballero, M., Davies, S.J., 2000, Records of Late Pleistocene-Holocene climatic change in Mexico - a review: Quaternary Science Reviews, 19, 699-721.

Metcalfe, S., Davies S., 2007, Deciphering recent climate change in central Mexican lake records: Climatic Change, 83, 169-186.

Mix, A.C., Bard, E., Schneider, R., 2001, Environmental processes of the ice age: land, oceans, glaciers (EPILOG): Quaternary Science Reviews, 20, 627-657.

Ohmura, A., Kasser, P., Funk, M., 1992, Climate at the equilibrium line of glaciers: Journal of Glaciology, 38, 397-411.

Ohngemach, D., Straka, H., 1983, Resumen en Español del trabajo en Puebla de la comunidad alemana, en Lauer, W. (ed.), Das MexicoProjeckt der Deutschen Forschungsgemeinschaft 18: Beiträge zur Vegetation- und Klimageschichte im Bebiet von Puebla - Tlaxcala: Wiesbaden, Hesse, Alemania, Steiner: 143-161.

Ortega, B., Caballero, C., Lozano, S., Israde, I., Vilaclara, G., 2002, 52000 years of environmental history in Zacapu basin, Michoacan, Mexico: the magnetic record: Earth and Planetary Science Letters, 202, 663-675.

Ortega, B., Vázquez, G., Caballero, M., Israde, I., Lozano-García, S., Schaaf, P., Torres, E., 2010, Late Pleistocene-Holocene record of environmental changes in Lake Zirahuen, central Mexico: Journal of Paleolimnology, 44, 745-760. 
Ramírez Nava, M., 2002, Cambios paleolimnológicos en el lago de Texcoco durante los últimos $c a$. 34000 años con base en el análisis de diatomeas: México D. F., Universidad Nacional Autónoma de México, Tesis de licenciatura, $43 \mathrm{p}$.

Reimer, P.J., Baillie, M.G.L., Bard, E., Bayliss, A., Beck, J.W., Bertrand, C., Blackwell, P.G., Buck, C.E., Burr, G., Cutler, K.B., Damon, P.E., Edwards, R.L., Fairbanks, R.G., Friedrich, M., Guilderson, T.P., Hughen, K. A., Kromer, B., McCormac, F.G., Manning, S., Bronk Ramsey, C., Reimer, R.W., Remmele, S., Southon, J.R., Stuiver, M., Talamo, S., Taylor, F.W., Plicht, J.V.D., Weyhenmeyer, C.E., 2004, IntCa104 terrestrial radiocarbon age calibration, 0-26 Cal kyr BP: Radiocarbon, 46, 1029-1058.

Rind, D., Peteet, D., 1985, Terrestrial conditions at the Last Glacial Maximum and CLIMAP sea-surface temperature estimates: Are they consistent?: Quaternary Research, 24, 1-22.

Roy, P.D., Caballero, M., Lozano, R., Pi, T., Morton, O., 2009, Late Pleistocene-Holocene geochemical history inferred from Lake Tecocomulco sediments, Basin of Mexico, Mexico: Geochemical Journal, 43, 49-64.

Sandoval Montaño, A., 2000, Análisis palinológico y consideraciones paleoambientales de un sondeo en el exlago de Texcoco, Cuenca de México: México, D. F., Universidad Nacional Autónoma de México, Tesis de maestría,. $195 \mathrm{p}$

Sedov, S., Solleiro-Rebolledo, E., Gama-Castro, J.E., Vallejo-Gómez, E., González-Velázquez, A., 2001, Buried paleosols of Nevado de Toluca: an alterative record of Late Quaternary environmental change in Central Mexico: Journal of Quaternary Science, 16, 375-389.

Sedov, S.N., Solleiro-Rebolledo, E., Gama-Castro, J.E., 2003a, Andosol to Luvisol evolution in Central Mexico: timing, mechanisms and environmental setting: Catena, 54, 495-513.

Sedov, S., Solleiro-Rebolledo, E., Morales-Puente, P., Arias-Herrería, A., Vallejo-Gómez, E., Jasso-Castañeda, C., 2003b, Mineral and organic components of the buried paleosols of the Nevado de Toluca, Central Mexico as indicators of paleoenvironments and soil evolution: Quaternary International, 106-107, 169-184.

Sedov, S, Solleiro-Rebolledo, S., Terhorst, B., Solé, J., Flores-Delgadillo, M.L., Werner, G., Poetsch, T., 2009a, The Tlaxcala basin paleosol sequence: A multiscale proxy of middle to late Quaternary environmental change in central Mexico: Revista Mexicana de Ciencias Geológicas, 26, 2, 448-465.

Sedov, S., Lozano-García, S., Solleiro-Rebolledo, E., McClung de Tapia, E., Ortega-Guerrero, B., Sosa-Nájera, S., 2009b, Tepexpan Revisited: A multiple proxy of local environmental changes in relation to human occupation from a paleolake shore section in central Mexico: Geomorphology, 122, 309-322.

Solleiro-Rebolledo, E., Sedov, S., McClung de Tapia, E., Cabadas, H., Gama-Castro, J., Vallejo-Gómez, E., 2006, Spatial variability of environment change in the Teotihuacan valley during late Quaternary: paleopedological inferences: Quaternary International, 156-157, 13-31.

Sosa Nájera, M.S., 2001, Registro palinológico del Pleistoceno tardíoHoloceno en el extremo meridional de la cuenca de México: paleoambientes e inferencias paleoclimáticas: México, D. F., Universidad Nacional Autónoma de México, Tesis de maestría, $115 \mathrm{p}$.
Straka, H., Ohngemach, D., 1989, Late Quaternary vegetation history of the Mexican highland: Plant Systematics and Evolution, 162: 115-132.

Torres Rodríguez, E., 2010, Evidencias de cambio climático durante el Pleistoceno-Holoceno en la región occidental de México a través del análisis palinológico en sedimentos del lago de Zirahuén, Michoacán: México, D. F., Universidad Nacional Autónoma de México, Tesis de Maestria, $116 \mathrm{p}$.

Vázquez-Selem, L., 2000, Late Quaternary glacial chronology of Iztaccíhuatl volcano, central Mexico. A record of environmental change in the border of the tropics: Tempe, Arizona, Arizona State University, Tesis doctoral, $210 \mathrm{p}$.

Vázquez-Selem, L., Heine, K., 2004, Late Quaternary glaciation of México, en Ehlers, J., Gibbard, P.L. (eds.), Quaternary Glaciations - Extent and Chronology, Volume 2, Part III: South America, Asia, Africa, Australia, Antarctica: Amsterdam, Países Bajos, Elsevier, 233-242.

Velázquez-Durán, R., 2003, Evolución paleoambiental del lago de Cuitzeo durante el Pleistoceno tardío: Historia de la vegetación e implicaciones paleoclimáticas: Morelia, Michoacán, México, Universidad Michoacana de San Nicolás de Hidalgo, Tesis de Maestría, 210 p.

Velázquez, A., Toledo, V.M., Luna, I., 2000, Mexican temperate vegetation, en Barbour, M.G., Dwight Billings, W., (eds.), North American Terrestrial Vegetation: Nueva York, Cambridge University Press, 574-592.

Watts, W.A., Bradbury, J.L., 1982, Paleoecological studies at Lake Patzcuaro on the west-central Mexican Plateau and at Chalco in the basin of Mexico: Quaternary Research, 17, 56-70.

Weninger, B., Jöris, O., 2008, $\mathrm{A}^{14} \mathrm{C}$ age calibration curve for the last 60 ka: the Greenland-Hulu U/Th timescale and its impact on understanding the Middle to Upper Paleolithic transition in Western Eurasia: Journal of Human Evolution, 55, 772-781.

White, S.E., 1962a, El Iztaccíhuatl. Acontecimientos volcánicos y geomorfológicos en el lado oeste durante el Pleistoceno Superior: México, D.F., Instituto Nacional de Antropología e Historia, $80 \mathrm{p}$.

White, S.E., 1962b, Late Pleistocene glacial sequence for the west side of Iztaccihuatl, Mexico: Geological Society of America Bulletin, 73, 935-958.

White, S.E., 1986, Quaternary glacial stratigraphy and chronology of Mexico: Quaternary Science Reviews, 5, 201-205.

White, S.E., Valastro, S., 1984, Pleistocene Glaciation of volcano Ajusco, central Mexico, and comparison with the standard Mexican glacial sequence: Quaternary Research, 21, 21-35.

White, S.E., Reyes-Cortes, M., Ortega Ramírez, J., Valastro, S., 1990, El Ajusco: geomorfología volcánica y acontecimientos glaciales durante el Pleistoceno superior y comparación con las series glaciales mexicanas y las de las Montanas Rocallosas: México, D. F., Instituto Nacional de Antropología e Historia, $77 \mathrm{p}$.

Manuscrito recibido: Octubre 13, 2009.

Manuscrito corregido recibido: Mayo 7, 2010.

Manuscrito aceptado: Mayo 24, 2010. 